Collection SFN 10 (2010) 605-630

(C) Owned by the authors, published by EDP Sciences, 2010

DOI: $10.1051 / \mathrm{sfn} / 2010016$

\title{
Utilisation de la diffusion des neutrons dans l'étude des mécanismes microscopiques à l'origine de la supraconductivité
}

\author{
S. Pailhès \\ Laboratoire Léon Brillouin, CE-Saclay, 91190 Gif-sur-Yvette, France
}

\begin{abstract}
Résumé. La supraconductivité non conventionnelle est certainement l'un des phénomènes les plus remarquables associés à la physique des systèmes dits à fortes corrélations électroniques. Dans ces systèmes, les degrés de liberté du spin et de la charge de l'électron sont très fortement couplés, de sorte que le déplacement des charges est contraint par l'environnement magnétique formé par leurs spins. La diffusion inélastique des neutrons est l'unique sonde expérimentale permettant de photographier l'état magnétique de ces systèmes dans tout l'espace réciproque et de suivre son évolution sur une échelle d'énergie parfaitement adaptée à celle de la supraconductivité. A travers l'exemple des cuprates supraconducteurs à haute température critique, nous montrons comment la diffusion inélastique des neutrons apporte des informations uniques permettant de comprendre l'organisation électronique de ces systémes à l'échelle microscopique et les mécanismes mise en jeu dans l'apparition de la supraconductivté.
\end{abstract}

\section{INTRODUCTION AUX CUPRATES SUPRACONDUCTEURS À HAUTE TEMPÉRATURE CRITIQUE}

Cette partie constitue une introduction succincte à la physique des cuprates supraconducteurs, essentiellement sur la base des observations faites par diffusion des neutrons. Le lecteur pourra trouver des introductions plus complètes dans les références suivantes en français $[21,28,57,66]$ et en anglais $[2,35,43,49,63]$.

La supraconductivité se caractérise par la disparition de toute résistance électrique (conductivité infinie) en dessous d'une température critique notée $T_{c}$ ainsi que par une réponse magnétique bien particulière : lorsqu'un supraconducteur est soumis à un champ magnétique, il expulse totalement les lignes de champ magnétique (diamagnétisme parfait). Cet effet, découvert par Meissner en 1933, démontre que les supraconducteurs ne sont pas uniquement des "super" conducteurs. L'idée d'une organisation cohérente des électrons à l'échelle macroscopique, appellée condensat supraconducteur, fut alors émise par London pour expliquer l'existence de super-courants à l'origine du comportement diamagnétique. La difficulté fut alors de comprendre comment ce condensat pouvait se former dans un métal. L'objectif des recherches dans ce domaine est de développer des approches instrumentales permettant de caractériser l'état microscopique de la phase supraconductrice et d'identifier l'interaction responsable de la mise en place de ce condensat et par suite de la phase supraconductrice.

\subsection{Une phase supraconductrice proche d'un état isolant antiferromagnétique}

Les cuprates supraconducteurs présentent une structure commune de type pérovskite caractérisée par l'empilement d'un ou plusieurs plans $\mathrm{CuO}_{2}$ séparés par d'autres couches atomiques jouant le rôle de réservoirs de charges (voir Fig. 1). Par transfert d'électrons entre les plans $\mathrm{CuO}_{2}$ et les réservoirs de

This is an Open Access article distributed under the terms of the Creative Commons Attribution-Noncommercial License 3.0, which permits unrestricted use, distribution, and reproduction in any noncommercial medium, provided the original work is properly cited. 

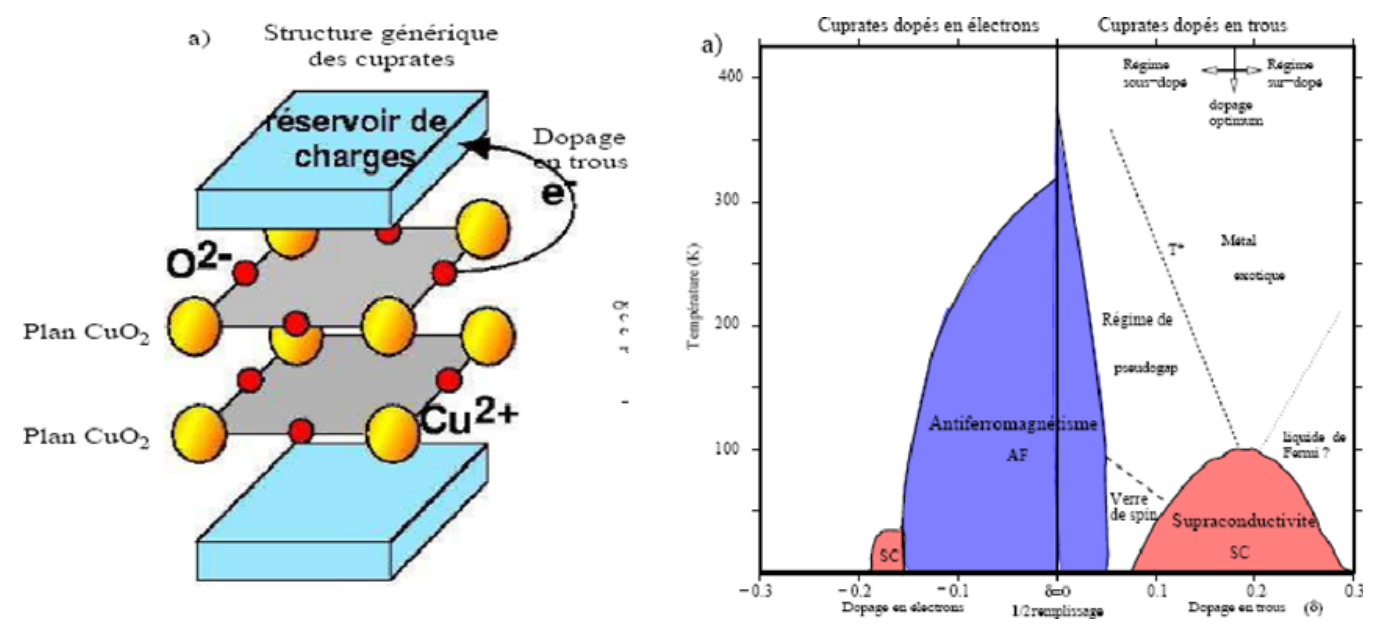

Figure 1. (a) Structure cristallographique simplifiée et (b) diagramme de phases générique des cuprates supraconducteurs dopés en électrons et en trous.

charges, il est possible de modifier la densité électronique dans les plans $\mathrm{CuO}_{2}$ : on parle de dopage en trous ou en électrons. En fonction du taux de dopage, les cuprates présentent un diagramme de phases générique présenté sur la Fig. 1. En l'absence de dopage, chaque ion $\mathrm{Cu}^{2+}$ ([Ar] $\left.3 \mathrm{~d}^{9}\right)$ possède un électron non apparié sur l'orbitale $3 \mathrm{~d}_{x^{2}-y^{2}}$. En termes de théorie des bandes, la bande du cuivre étant demie-remplie, le système devrait donc être métallique à dopage nul. Au contraire, ces matériaux sont isolants. Les spins $\mathrm{S}=1 / 2$ sur les sites de cuivre s'ordonnent antiferromagnétiquement au dessous de la température dite de Néel $\left(T_{N} \sim 300-400 \mathrm{~K}\right)$. La structure correspondante, caractérisé par diffusion inélastique des neutrons, est bien décrite par un modèle de Heisenberg appliqué à un système de spins $(\mathrm{S}=1 / 2)$ quantique $[29,61]$. Dans sa forme la plus simple, le Hamiltonien d'Heisenberg s'écrit :

$$
H_{H}=J \sum_{\langle i, j\rangle} \vec{S}_{i} \cdot \vec{S}_{j}
$$

Nous montrons sur la Fig. 2 une mesure des ondes de spins dans le cuprate $\mathrm{La}_{2} \mathrm{CuO}_{4}$ sur un spectromètre à temps de vol [15]. Sur la figure de gauche sont reportées (A) les coupes à énergie constante et (B) les coupes en énergie pour plusieurs vecteurs d'ondes à travers la nappe de dispersion des ondes de spins. La dispersion expérimentale est présentée sur la figure de droite, selon les directions de symétrie principales dans l'espace réciproque. Les lignes noires correspondent à une modélisation obtenue en dérivant une forme plus complexe de l'Hamiltonien d'Heisenberg (Eq. (1.1)) incluant des termes d'échanges magnétiques entre seconds et troisièmes plus proches voisins, ainsi qu'un terme d'échange circulaire [15].

L'état isolant des cuprates résulte de la forte répulsion coulombienne intra-site, $U$, qui interdit la double occupation sur le site du cuivre. Typiquement $U \sim 2 \mathrm{eV}$ alors que le terme de saut entre les sites de cuivre plus proches voisins est $t \sim 0.3 \mathrm{eV}$ conduisant à des valeurs de super-échange $J=4 t^{2} / U$ de l'ordre de $0.13 \mathrm{eV}$, en bon accord avec les mesures expérimentales. Les oxydes de cuivre se trouvent donc dans un régime de couplage intermédiaire où $U \sim W=4 t$ ( $W$ étant la largeur de bande) où les charges ne sont ni complétement localisées, ni totalement libres de se mouvoir.

\subsection{Un état microscopique complexe et encore incompris dans le régime métallique}

Les trous sont introduits par dopage au niveau des orbitales $2 \mathrm{p}_{\sigma}$ des oxygènes du plan de base. Le recouvrement des orbitales $2 \mathrm{p}_{\sigma}$ de l'oxygène et des orbitales $3 \mathrm{~d}_{x^{2}-y^{2}}$ du cuivre offre la possibilité 

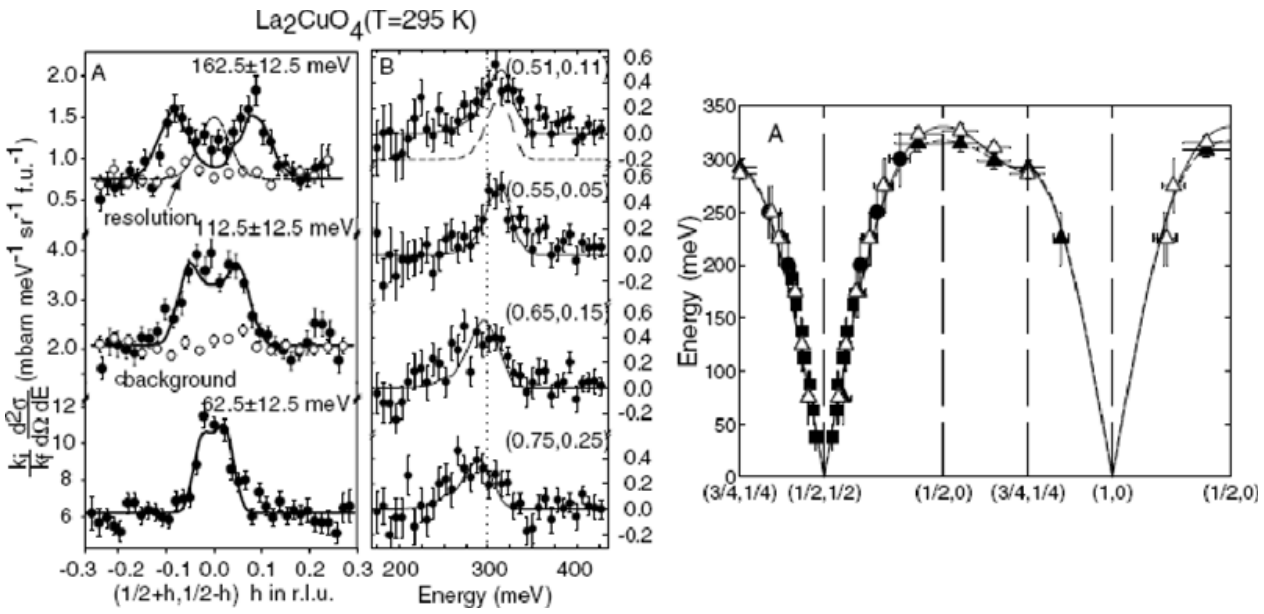

Figure 2. Mesure neutron des ondes de spins dans le cuprate $\mathrm{La}_{2} \mathrm{CuO}_{4}$ à $\mathrm{T}=295 \mathrm{~K}$ [15]. (gauche) (A) Coupes à énergie constante autour du vecteur d'onde $\mathrm{AF}$ et $\mathrm{B}$ ) coupe en énergie pour différentes valeurs de vecteurs d'ondes. Les positions des maxima permettent de caractériser la dispersion des ondes de spins. Les lignes noires correspondent à la convolution entre un modèle d'onde de spin d'Heisenberg et la fonction de résolution instrumentale, indiquée sur la première figure de A. (droite) Relation de dispersion des ondes de spins obtenue selon différentes directions de symétrie dans la zone de Brillouin. Les points avec des barres d'erreurs verticales correspondent aux maxima des coupes à énergie constante et les autres aux maxima des coupes à vecteur d'onde constant. La ligne correspond à la dispersion calculée en utilisant le même modèle d'Heisenberg.
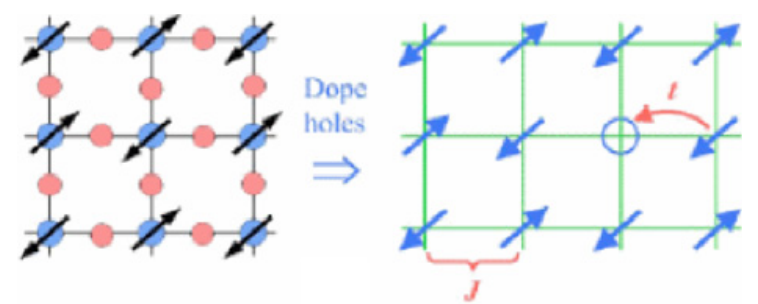

Figure 3. Dopage en trous d'un isolant de Mott [43]. (gauche) Description schématique du plan $\mathrm{CuO}_{2}$ (ions $\mathrm{Cu}^{2+}$ en bleu et $\mathrm{O}^{2-}$ en rouge). Les liaisons $\mathrm{Cu}-\mathrm{O}$ ainsi que les spin sont représentés respectivement par des traits continus noirs et des flèches noires. $J$ indique le couplage de super-échange entre les spins du cuivre. Le plan $\mathrm{CuO}_{2}$ peut être vu comme un réseau plan carré pavé de plaquettes $\mathrm{CuO}_{2}$. Ces plaquettes peuvent être dans trois états : $|\uparrow\rangle$ et $|\downarrow\rangle$ lorsque la plaquette ne contient pas de trous de dopage, $|0\rangle$ lorsqu'il y a un trou de dopage (état singulet de Zhang et Rice [71]). (droite) Le mouvement d'un trou d'une plaquette à une autre revient à retouner le spin d'une plaquette.

au trou de se déplacer par saut d'un site de cuivre à l'autre. La modélisation théorique des cuprates devrait donc faire intervenir un Hamiltonien à 3 bandes incluant les orbitales $d$ du cuivre et $p$ des oxygènes. Cependant, Zhang et Rice (ZR) ont proposé [71] qu'un trou de dopage sur le site de l'oxygène puisse former un état singulet avec l'electron sur le site du cuivre. Ce singulet a donc trois états de spins possibles : $|\uparrow\rangle$ et $|\downarrow\rangle$ lorsque la plaquette ne contient pas de trous de dopage, $|0\rangle$ lorsque le singulet ZR est formé. De cette manière, il est possible de considérer un modèle d'Hubbard à une seule bande avec une intégrale de saut effective $t=t_{p d}^{2} /\left(E_{p}-E_{d}\right)$ et une interaction coulombienne effective $U=E_{p}-E_{d}$. Dans la limite réaliste pour les cuprates de $U \gg t$, l'Hamiltonien prend la forme de celui 
du modèle t-J $[16,43]$ :

$$
H=-\sum_{i, j, \sigma} t_{i j} \widetilde{c}_{i, \sigma}^{\dagger} \widetilde{c}_{j, \sigma}+\sum_{i, j}\left(J \vec{S}_{i} \vec{S}_{j}-\frac{n_{i} n_{j}}{4}\right)
$$

Les opérateurs $\widetilde{c}_{i, \sigma}=c_{i, \sigma}\left(1-n_{i,-\sigma}\right)$ permettent de décrire le saut des trous de dopage d'un site à un autre tout en respectant l'interdiction de double occupation des sites de cuivre. $J$ est l'intégrale de superéchange $\mathrm{AF}$ égal à $4 t^{2} / \mathrm{U}=4 t_{p d}^{4} /\left(E_{d}-E_{p}\right)^{3}$.

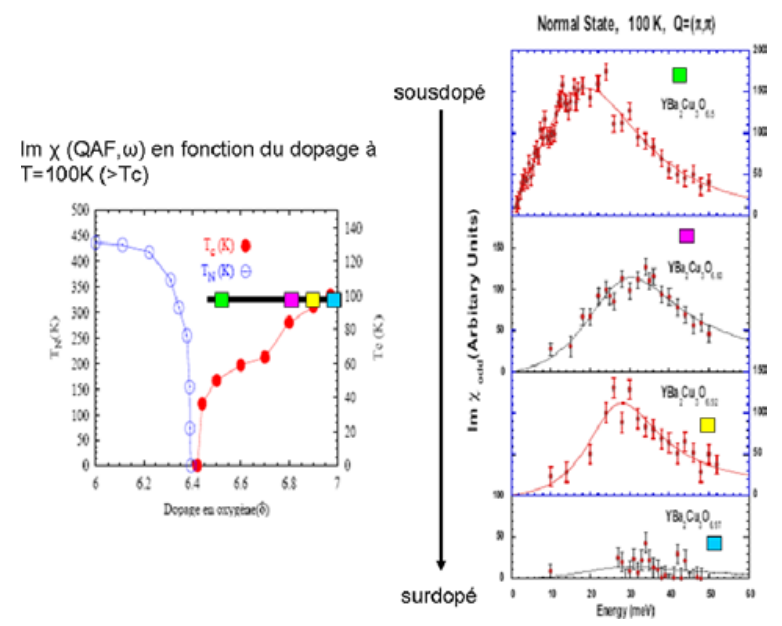

Figure 4. Evolution de la susceptibilité magnétique au vecteur d'onde antiferromagnétique en fonction du dopage dans le cuprate $\mathrm{YBa}_{2} \mathrm{Cu}_{3} \mathrm{O}_{6+x}$ mesurée par neutron. [5,6]. (gauche) Diagramme de phases du cuprate $\mathrm{YBa}_{2} \mathrm{Cu}_{3} \mathrm{O}_{6+x}$ en fonction du taux d'oxygène $\mathrm{x}$. L'oxygénation de la structure permet d'augmenter le dopage en trous des plans $\mathrm{CuO}_{2}$. Les $\mathrm{T}_{N}$ sont reportées sur l'axe des ordonnées de gauche et les $\mathrm{T}_{c}$ sur celui de droite. (droite) Mesures neutrons de la partie imaginaire de la susceptibilité magnétique en fonction de l'énergie au vecteur d'onde antiferromagnétique $\operatorname{Im} \chi\left(Q_{A F}, \omega\right)$. Les mesures ont été réalisées à différents dopages indiqués sur la figure de gauche.

Le singulet de ZR étant dans une matrice de spins couplés AF, son mouvement implique le renversement d'un spin et conduit donc à une frustration de la matrice antiferromagnétique (voir Fig. 3). L'état AF de Néel est rapidement détruit pour des faibles dopages en trous de l'ordre de 2-4\%. Pour des dopages supérieurs, le système devient métallique et supraconducteur. Dans le diagramme de phase, la supraconductivité forme un dôme symétrique de part et d'autre du dopage optimal où la valeur de la $\mathrm{T}_{c}$ est maximale. L'état «normal» au-dessus du dôme supraconducteur est généralement divisé en 2 régions correspondant à des régimes métalliques différents (Figure 1) : le régime sous-dopé proche de la transition isolant/métal et le régime sur-dopé. En effet, bien que l'ordre $\mathrm{AF}$ soit détruit à la transition isolant/métal, il persiste de fortes fluctuations antiferromagnétiques dans le spectre magnétique. Celles-ci sont mises en évidence par diffusion inélastique des neutrons, qui comme nous l'avons vu dans le cours de F. Moussa, donne une information directe sur la dépendance en énergie de la partie imaginaire de la susceptibilité magnétique, $\operatorname{Im} \chi\left(Q_{A F}, \omega\right)$. La Figure 4 présente les mesures neutrons de $\operatorname{Im} \chi\left(Q_{A F}, \omega\right)$ dans l'état normal pour différentes valeurs de dopages en trous dans le cuprate $\mathrm{YBa}_{2} \mathrm{Cu}_{3} \mathrm{O}_{6+x}$ [5]. Dans le régime sous-dopé, $\operatorname{Im} \chi\left(Q_{A F}, \omega\right)$ présente un pic intense, large en énergie et asymétrique, dont la position du maximum d'intensité se situe autour de $15 \mathrm{meV}$ dans l'échantillon le plus sous-dopé et se déplace à plus haute énergie en augmentant le dopage. 


\subsubsection{Le régime sur-dopé}

A mesure que le dopage augmente, l'intensité du pic diminue jusqu'à devenir non détectable au dopage optimal, ce qui correspond à un régime métallique classique. Ainsi, dans le régime sur-dopé, la description microscopique de l'état métallique correspond à celle d'un liquide de Fermi: quasiparticules bien définies sur toute la surface de Fermi, dépendance quadratique de la résistivité en fonction de la température, dépendance linéaire de la chaleur spécifique électronique en fonction de la température [67], etc ... Il est alors utile de considérer la limite dite de couplage faible, $\mathrm{U} \ll \mathrm{t}$, de l'Hamiltonien d'Hubbard à une seule bande $[42,46]$ :

$$
H=-\sum_{i, j, \sigma} t_{i j} c_{i, \sigma}^{\dagger} c_{j, \sigma}+U \sum_{i} n_{i, \sigma} n_{i,-\sigma}
$$

L'interaction U est alors traitée en perturbation en utilisant l'approximation RPA. L'expression de la susceptibilité magnétique est :

$$
\chi_{R P A}=\frac{\chi_{0}}{1-U \chi_{0}}
$$

où $\chi_{0}$ est la susceptibilité magnétique sans interaction. Comme nous le verrons ci-dessous, $\chi_{0}$ et donc $\chi_{R P A}$ peuvent être totalement déterminées en mesurant la nappe de dispersion des énergies des états de quasiparticules obtenue par photoémission résolue en angle.

\subsubsection{Le régime sous-dopé}

La description microscopique de l'état métallique dans le régime sous-dopé est plus délicate et fait encore l'objet de vigoureux débats. En plus des fortes fluctuations antiferromagnétiques, toutes les sondes microscopiques et macroscopiques révèlent de nombreuses déviations par rapport aux comportements usuels du liquide de Fermi [37,68]. Il apparaît notamment en dessous de $T^{*}$ un «pseudo-gap»dans le spectre des excitations magnétiques et de charges. Le lecteur trouvera dans les références toute la phénoménologie de la phase de pseudo-gap. La complexité du système dans ce régime est telle qu'il a été finalement proposé que ce régime métallique étrange puisse être une phase ordonnée à longue portée associée à un nouvel état de la matière. La difficulté est alors de trouver le paramètre d'ordre qui lui est associé et de savoir s'il y a coexistence ou non avec la supraconductivité. D’un point de vue théorique, la difficulté réside dans les opérateurs $\widetilde{c}_{i, \sigma}$ de l'Eq. (1.2) qui représentent le déplacement du singulet de ZR en respectant l'interdiction de double occupation d'un site de cuivre (limite $U \gg t$ ). Cet opérateur et son complexe conjugué ne commutent pas et ne sont associés ni à la statistique de Fermi ni à celle de Bose-Einstein. Il existe ainsi plusieurs approximations théoriques conduisant à différents paramètres d'ordres possibles associés à la phase de pseudo-gap [52]. Nous citons ci-dessous les exemples de deux propositions théoriques présentant une signature magnétique bien spécifique.

\section{L'approche des «stripes » $[38,39]$}

Les calculs numériques sur de petits clusters indiquent une tendance très prononcée du système à la ségrégation de charges. Intuitivement, les trous ont tendance à s'agglomérer et à se déplacer ensemble pour minimiser le nombre de liens AF brisés. A cette tendance s'oppose la répulsion coulombienne entre charges, si bien que ces calculs montrent la formation de parois de discommensuration séparant des régions antiferromagnétiques : les «stripes». L'aimantation alternée propre à chaque domaine magnétique s'inverse au passage à travers ces parois. La présence de stripes suivant par exemple la direction $b$ est facilement identifiable par des mesures de diffraction de neutrons. Par couplage au réseau, l'ordre des charges donne naissance à des pics structuraux aux vecteurs d'ondes $\left(0, \pm 2 \delta_{\text {inc }}\right)$. L'ordre magnétique implique l'apparition de pics de sur-structure magnétique aux vecteurs d'ondes $\left(0.5,1 \pm \delta_{\text {inc }}, 0.5\right)$ (voir Fig. 5). 
La réalisation d'un état microscopique de type stripes a été observée par diffusion des neutrons dans les nickelates isolants et dans les cuprates ( $\mathrm{La}, \mathrm{Nd})_{2-x} \mathrm{Sr}_{x} \mathrm{CuO}_{4}$ [69] (voir Fig. 5) et $\mathrm{La}_{7 / 8} \mathrm{Ba}_{1 / 8} \mathrm{CuO}_{4}$ [27]. Le spectre des excitations magnétiques dans de tels systèmes est donc caractérisé par une forte anisotropie 1D. Malheureusement, celle-ci est masquée par le mâclage. Seul le cuprate $\mathrm{YBa}_{2} \mathrm{Cu}_{3} \mathrm{O}_{6+x}$ a pu être démâclé et étudié aux neutrons. Ces études ont écarté l'hypothèse de stripes statiques, mais il reste encore la possibilité de stripes fluctuants non ancrés sur le réseau cristallin. Par analogie avec les cristaux liquides, on parle d'état nématique. Toutefois, le débat sur l'existence d'une ségrégation de phases dans les cuprates de type stripes n'est pas encore clos.

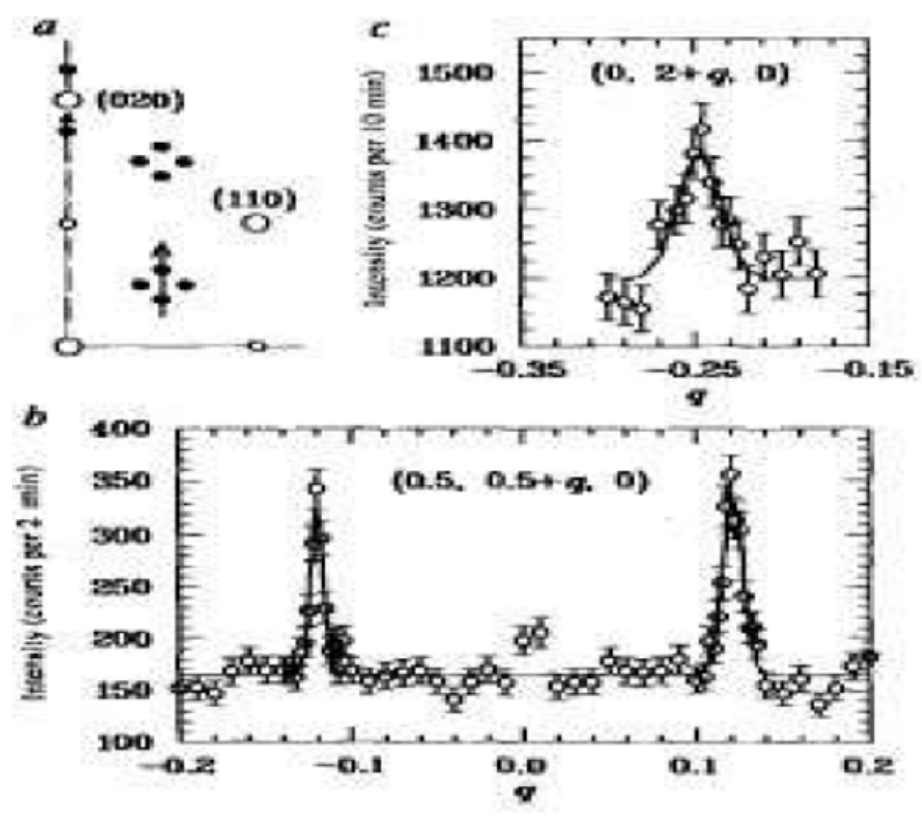

Figure 5. Ordre de stripes mis en évidence par diffusion des neutrons dans le cuprate non supraconducteur $\mathrm{La}_{1-x} \mathrm{Nd}_{0.4} \mathrm{Sr}_{x} \mathrm{CuO}_{4}(x=1 / 8)$ [69]. La figure a) est un schéma de la première zone de Brillouin. Les cercles pleins représentent les pics de Bragg associés à l'ordre des spins et de charge, et les cercles vides les pics de Bragg nucléaires. Les coupes en vecteur d'onde des figures c) et b) sont représentées par des flèches noires. Sur la figure c), on observe le pic de sur-structure nucléaire et sur la figure b) les pics de sur-structures magnétiques. Le système étant tétragonal, les stripes s'alignent indifféremment suivant les axes a et b, engendrant 4 spots magnétiques autour du vecteur d'onde AF et non uniquement 2.

\section{Les courants circulants}

D'autres modèles proposent l'existence de courants circulants (ou boucles de courants) à l'intérieur des plans $\mathrm{CuO}_{2}$. Ceux-ci pourraient être à l'origine de la phase de pseudo-gap. Il s'agit de la phase de flux proposée par Affleck et Marson, de la phase onde de densité de charges non conventionnelle de symétrie $d$ (notée DDW) proposée par S. Chakraverty, ainsi que des phases de courants circulants proposées par C. Varma. Les paramètres d'ordres associés aux deux premiers modèles brisent la symétrie de translation et de renversement du temps. En revanche, le paramètre d'ordre de C. Varma ne brise pas la symétrie de translation. Pour ce dernier, le signal associé aux boucles de courants se superpose aux pics de Bragg nucléaires. L'utilisation des neutrons polarisés en spin est alors essentielle pour extraire de façon sélective le signal magnétique généré par les courants. Sur la Fig. 6, nous montrons des résultats de neutrons polarisés mettant en évidence l'existence de telles boucles dans le cuprate $\mathrm{Y}_{1-y} \mathrm{Ca}_{y} \mathrm{Ba}_{2} \mathrm{Cu}_{3} \mathrm{O}_{6+x}$. Sur cette figure sont reportés les suivis en température des intensités brutes 
mesurées sur les positions des pics de Bragg $\vec{q}=(0,1,1)$ et $\vec{q}=(0,0,2)$. L'analyse de la polarisation des neutrons permet de distinguer la part du signal magnétique de la contribution nucléaire. Ainsi, ces mesures mettent en évidence l'amplification de l'intensité magnétique uniquement en dessous d'une certaine température $\mathrm{T}_{\text {mag }}$. Celle-ci diminue à mesure que le dopage augmente. De plus, $\mathrm{T}_{\text {mag }}$ décroît linéairement avec le dopage en trous et suit la ligne définissant $\mathrm{T}^{*}$ dans le diagramme de phases (voir figure de droite). Enfin, $\mathrm{T}_{\text {mag }}$ s'extrapole à température nulle pour un dopage en trous de 0.19, qui pourrait donc correspondre à un point critique quantique dans le diagramme de phase. Ces mesures neutrons prouvent l'existence d'un ordre «caché » associé à la phase de pseudo-gap. Là encore, le débat n'est pas clos, et certains désaccords persistent entre expériences et théorie.

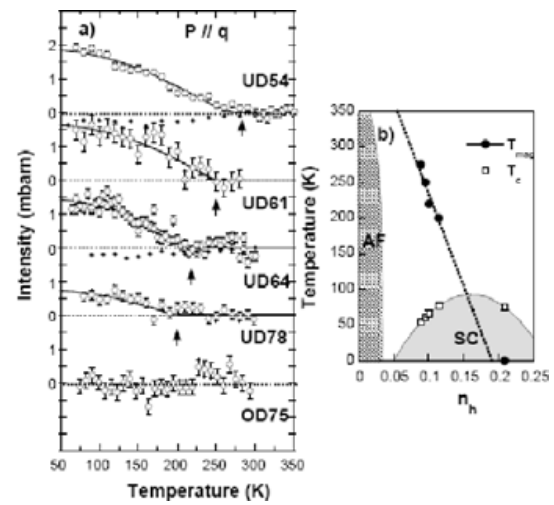

Figure 6. Observation neutrons de courants orbitaux dans le système $\mathrm{Y}_{1-y} \mathrm{Ca}_{y} \mathrm{Ba}_{2} \mathrm{Cu}_{3} \mathrm{O}_{6+x}$ [21,22]. (gauche) Dépendance en température des intensités magnétiques normalisées mesurées dans cinq échantillons à des dopages différents aux vecteurs d'ondes $\vec{q}=(0,1,1)$ (ronds vides) et $\vec{q}=(0,0,2)$ (ronds pleins). Il s'agit de mesures de neutrons polarisés permettant de séparer les contributions magnétiques (symboles ouverts) des contributions nucléaires (symboles pleins). Sur les deux pics de Bragg, l'intensité magnétique augmente en dessous d'une certaine température $\mathrm{T}_{\text {mag }}$, qui varie linéairement en fonction du dopage. (droite) Diagramme de phases de $\mathrm{Y}_{1-y} \mathrm{Ca}_{y} \mathrm{Ba}_{2} \mathrm{Cu}_{3} \mathrm{O}_{6+x}$. Les points correspondant aux températures $\mathrm{T}_{m a g}$ suivent la ligne de pseudo-gap.

\subsection{Quelques propositions sur l'origine microscopique de l'appariement supraconducteur}

La supraconductivité dans les cuprates est dite non conventionnelle. Cette dénomination est due aux valeurs exceptionnellement grandes de $\mathrm{T}_{c}$ qui peuvent atteindre $160 \mathrm{~K}$ et, surtout, à la symétrie du paramètre d'ordre supraconducteur. En effet, celui-ci est anisotrope et de symétrie « $d_{x^{2}-y^{2}}$ », c'està-dire caractérisée par un changement de signe de part et d'autre des lignes d'annulation (ou noeuds) à $45^{\circ}$ des liaisons $\mathrm{Cu}-\mathrm{O}$.

Nous venons de voir que l'organisation microscopique de l'état non supraconducteur des cuprates est encore sujette à des propositions théoriques très opposées. L'origine principale de cette divergence provient de l'importance accordée à la nature «fortement corrélée » des quasiparticules dans le système.

Dans les théories de couplage fort $(U \gg t)$, la physique pilotée par les fortes corrélations électroniques est à l'origine de la supraconductivité à haute température critique. L'état normal est très différent d'un liquide de Fermi et plusieurs types d'ordres peuvent être stabilisés : phase de stripes, séparation spin/charge, boucles de courants etc. La physique à l'origine de ces phases étranges est celle qui permet l'apparition de la supraconductivité. Le mécanisme d'appariement est alors différent du mécanisme BCS, qui nécessite un état normal décrit par des quasiparticules bien définies. En règle générale, dans les modèles de couplage fort, c'est l'interaction de super-échange $\mathrm{AF}, J(\vec{q})=$ $2 J\left(\cos \left(q_{x}\right)+\cos \left(q_{y}\right)\right)$, qui joue le rôle de l'interaction attractive entre charges. Les fluctuations $\mathrm{AF}$ jouent un rôle secondaire et, au mieux, assistent l'interaction directe. 
Dans la limite du couplage faible, l'état normal est considéré comme étant très proche d'un liquide de Fermi. La phase de pseudo-gap entre en compétition avec l'ordre supraconducteur. La base du mécanisme d'appariement des charges repose sur les théories BCS/Eliashberg. L'originalité provient alors de la nature de l'excitation qui véhicule l'appariement. Il peut notamment se faire par un échange de fluctuations magnétiques. Le potentiel de paires, $\mathrm{V}(\vec{q}, \omega)$, s'écrit :

$$
V(\vec{q}, \omega)=\frac{3}{2} U^{2} \chi(\vec{q}, \omega)
$$

où $\chi(\vec{q}, \omega)$ est la susceptibilité magnétique généralisée. Ainsi, le terme de répulsion coulombienne entre charges, $U$, participe à l'amplification de la réponse magnétique et donc à l'appariement supraconducteur.

Enfin, l'appariement via un couplage avec le réseau est très peu probable, mais possible. L'Hamiltonien du modèle $t$ - $J$, Eq. (1.2), est purement électronique et n'inclut pas de couplages électronphonon (el-ph). Néanmoins, ces couplages existent et sont mesurés [3]. Cependant, la symétrie $d$ du gap supraconducteur et l'absence d'effet isotopique quel que soit le taux de dopage sur la température de supraconduction sont les principaux obstacles à un appariement via un couplage el-ph. Concernant la symétrie, notons qu'il est possible de trouver certains phonons dont le couplage avec les électrons est de symétrie $d$. C'est le cas d'un mode de vibration de l'oxygène perpendiculaire aux plans $\mathrm{CuO}_{2}$. Pour ce mode, la constante de couplage est de la forme $g(\vec{q})=g_{0}^{2}\left(\cos ^{2} q_{x} / 2+\cos ^{2} q_{y} / 2\right)$ [10,39]. L'absence d'effet isotopique à tout dopage est plus difficile à concevoir, même s'il peut être expliqué dans certains modèles.

\section{EXCITATIONS MAGNÉTIQUES NON CONVENTIONNELLES DANS L'ÉTAT SUPRACONDUCTEUR DES CUPRATES MESURÉES PAR NEUTRONS}

Dans cette partie, nous nous concentrons sur les expériences neutrons qui mettent en lumière l'apparition d'une "résonance", correspondant à une excitation triplet de spin $\mathrm{S}=1$ au dessous de $\mathrm{T}_{c}$.

\subsection{La section de diffusion inélastique magnétique dans les cuprates}

Dans le cours de F. Moussa, nous avons relié la section efficace différentielle neutron à la transformée de Fourier dans l'espace et le temps de la fonction de corrélation spin-spin. Soit, pour un faisceau de neutrons incidents non polarisés :

$$
\frac{d^{2} \sigma}{d \Omega d \omega}=\left(\gamma r_{o}\right)^{2} \frac{k^{\prime}}{k}\left|f(\vec{Q})_{C u^{2+}}\right|^{2} \exp (-2 W(\vec{Q})) \sum_{\alpha, \beta=x, y, z}\left(\delta_{\alpha \beta}-\frac{Q^{\alpha} Q^{\beta}}{Q^{2}}\right) S^{\alpha \beta}(\vec{Q}, \omega)
$$

où $k^{\prime}$ et $k$ sont les vecteurs d'ondes incidents et diffusés des neutrons, $r_{o}^{2}=\left(\frac{e^{2}}{m_{e} c^{2}}\right)^{2}=0.29$ barn, $f(\vec{q})_{C u^{2+}}$ est le facteur de forme magnétique des orbitales $\mathrm{d}_{x^{2}-y^{2}}$ de l'ion $\mathrm{Cu}^{2+}$. Les comportements en vecteur d'onde des signaux magnétiques dans les cuprates (ondes de spin, modes magnétiques résonnants, etc...) évoluent comme le facteur de forme magnétique des orbitales $\mathrm{d}_{x^{2}-y^{2}}$ de l'ion $\mathrm{Cu}^{2+}[5,65]$. Ce facteur est anisotrope dans le plan de base et son intensité diminue à mesure que l'amplitude du vecteur d'onde transféré augmente. C'est une propriété importante de tout signal magnétique mesuré par neutrons : contrairement à un signal d'origine nucléaire, l'intensité magnétique décroît pour les grandes valeurs de $|\vec{Q}|$. Cette propriété est souvent utilisée dans les expériences de diffusion des neutrons non polarisés afin de prouver l'origine magnétique du signal. Ainsi, si possible, les mesures neutrons d'excitations magnétiques se font aux petits vecteurs d'ondes. Enfin, $\mathrm{W}(\vec{Q})$ est le facteur de Debye-Waller qui est fonction du déplacement de l'atome autour de sa position d'origine. Celui-ci est 
généralement négligé car les déplacements moyens des atomes de cuivre sont de l'ordre du millième d'Angstrom [64] sur la gamme de température $10 \mathrm{~K}-300 \mathrm{~K}$.

La section efficace différentielle neutron est donc directement proportionnelle à la transformée de Fourier dans l'espace et dans le temps de la fonction de corrélation spin-spin $S^{\alpha \beta}(\vec{Q}, \omega)$, qui s'écrit [44] :

$$
S^{\alpha \beta}(\vec{Q}, \omega)=\frac{1}{2 \pi} \sum_{i, j} \exp \left(-i \vec{Q} \cdot\left(\vec{R}_{i}-\vec{R}_{j}\right)\right) \int d t\left\langle S_{i}^{\alpha}(0) S_{j}^{\beta}(t)\right\rangle \exp (-i \omega t)
$$

Dans le cadre de la théorie de la réponse linéaire, on démontre que la fonction de corrélation de spin du système est directement proportionnelle à la partie imaginaire de la susceptibilité généralisée [44], $\chi(\vec{q}, \omega)=\chi^{\prime}(\vec{q}, \omega)+i \chi^{\prime \prime}(\vec{q}, \omega)$ :

$$
S^{\alpha \beta}(\vec{Q}, \omega)=\frac{1+n(\omega, T)}{\pi} \frac{\chi_{\alpha, \beta}^{\prime \prime}(\vec{q}, \omega)}{\left(g \mu_{B}\right)^{2}}
$$

$\chi_{\alpha, \beta}^{\prime \prime}(\vec{q}, \omega)$, est exprimé en $\mu_{B}^{2} \cdot e V^{-1} \cdot\{1+n(\omega, T)\}=\frac{1}{1-\exp \left(\frac{-\hbar \omega}{k_{B} T}\right)}$, est le facteur du "bilan détaillé" (voir le cours de H. Schober).

Dans les cuprates, on considère généralement le cas isotrope dans l'espace des spins : il n'y a donc pas de direction privilégiée des fluctuations magnétiques, soit $\chi_{x x}^{\prime \prime}(\vec{q}, \omega)=\chi_{y y}^{\prime \prime}(\vec{q}, \omega)=\chi_{z z}^{\prime \prime}(\vec{q}, \omega)$, le facteur d'orientation vaut 2. La section efficace de diffusion se simplifie :

$$
\frac{d^{2} \sigma}{d \Omega d \omega}=\frac{2 r_{0}^{2}}{\pi} \frac{k_{f}}{k_{i}}\left|f(\vec{Q})_{C u^{2+}}\right|^{2} \frac{\chi^{\prime \prime}(\vec{q}, \omega)}{\left(g \mu_{B}\right)^{2}}
$$

La susceptibilité longitudinale, $\chi^{\prime \prime}(\vec{q}, \omega)$, est exprimée en $\mu_{B}^{2} \cdot e V^{-1} \cdot f \cdot u^{-1}$ («f.u.»formula unit). En général, dans les travaux théoriques, on trouve plutôt la susceptibilité transverse $\chi_{+-}^{\prime \prime}(\vec{q}, \omega)$ qui est égale à 2 fois la susceptibilité longitudinale.

\subsection{Les échantillons}

La diffusion inélastique des neutrons pour l'étude des fluctuations magnétiques dans les cuprates nécessite l'utilisation de monocristaux d'un volume minimum de l'ordre de $0.1 \mathrm{~cm}^{3}$ pour un spectromètre 3-axes et de l'ordre de plusieurs $\mathrm{cm}^{3}$ pour un spectromètre à temps de vol. De tels volumes monocristallins sont accessibles uniquement dans deux familles de cuprate, $\mathrm{La}_{2}-\mathbf{x} \mathrm{Sr}_{\mathbf{x}} \mathrm{Cu}_{\mathbf{4}} \mathrm{O}_{4}$
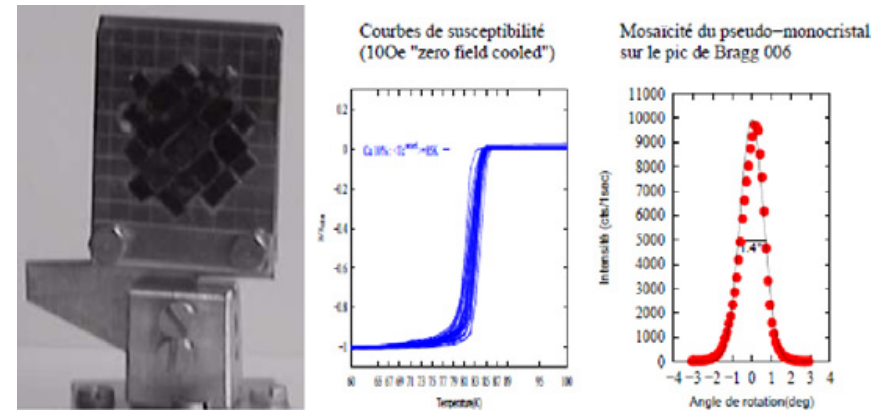

Figure 7. Pseudo-monocristal de $\mathrm{Y}_{0.9} \mathrm{Ca}_{0.1} \mathrm{Ba}_{2} \mathrm{Cu}_{3} \mathrm{O}_{7-\delta}(M \sim 2 g)$ pour une mesure de diffusion inélastique des neutrons sur spectromètre 3-axes du LLB ou de l'ILL [57,59]. (gauche) Photo du montage correspondant à un co-alignement de 60 échantillons. (gauche) Courbes de susceptibilité magnétique de l'ensemble des petits monocristaux participant au montage. (droite) Mesure d'un pic de Bragg nucléaire (raie 006) sur ce pseudomonocristal. La mosaïcité globale est de 1.4 degré. 


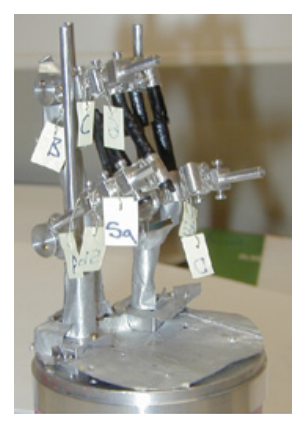

Figure 8. Pseudo-monocristal de $\mathrm{La}_{2-x} \mathrm{Sr}_{x} \mathrm{Cu}_{4} \mathrm{O}_{4}(M \sim 20 g)$ pour une mesure de diffusion inélastique des neutrons sur le spectromètre à temps de vol MAPS à ISIS [13].

et $\mathrm{YBa}_{2} \mathrm{Cu}_{3} \mathrm{O}_{6+\mathbf{x}}$. Récemment les études de diffusion inélastique des neutrons ont pu s'ouvrir à d'autres familles de cuprates, en reconstituant un «pseudo-monocristal»à partir d'un grand nombre de monocristaux de volume individuel bien inférieur à $0.1 \mathrm{~cm}^{3}$. Ce procédé a été mis en pratique et son efficacité a été démontrée par les études sur pseudo-monocristaux de $\mathrm{Tl}_{2} \mathrm{Ba}_{2} \mathrm{CuO}_{6+\mathbf{d}}$ [30], $\mathrm{Y}_{1-x} \mathrm{Ca}_{x} \mathrm{Ba}_{2} \mathrm{Cu}_{3} \mathrm{O}_{6+\mathbf{x}}[59]$ et $\mathrm{BSCO}[12,22]$. Cette technique de montage permet également d'obtenir des monocristaux de qualité supérieure, augmentant ainsi la précision des expériences. En effet, les gros monocristaux de $\mathrm{YBa}_{2} \mathrm{Cu}_{3} \mathrm{O}_{6+\mathbf{x}}$, type pastille de $1-2 \mathrm{~cm}$ de diamètre, sont généralement de qualité cristallographique médiocre. Typiquement, la mosaïcité de ces gros monocristaux est de l'ordre de $3^{\circ}$. Aujourd'hui, en assemblant les petits monocristaux, il est possible d'atteindre des mosaïcités inférieures à $1.5^{\circ}$ pour le pseudo-monocristal. De plus, les effets de certains traitements (substitution, démâclage) uniquement réalisables ou contrôlés sur des monocristaux de petite taille peuvent maintenant être étudiés. C'est ainsi que des mesures de neutrons ont été menées sur des pseudo-monocristaux démâclés de $\mathrm{Y}_{1-x} \mathrm{Ca}_{x} \mathrm{Ba}_{2} \mathrm{Cu}_{3} \mathrm{O}_{6+\mathbf{x}}$ [32] constitués d'un ensemble de petits monocristaux individuellement démâclés. Ces mesures ont permis de répondre à la problématique de la dimensionnalité des fluctuations magnétiques observées dans les cuprates.

\subsection{Taille de la résolution expérimentale $v s$ taille de la physique}

Sur la Fig. 9, l'ellipsoïde de résolution calculé au vecteur d'onde $\mathrm{Q}_{A F}=(0.5,0.5,5.2)$ est représenté en fonction de l'énergie et du vecteur d'onde selon la direction 110. Les conditions de mesures, indiquées sur la figure sont usuelles. La ligne violette correspond à la limite cinématique obtenue à partir des équations de conservation de l'énergie et de la quantité de mouvement. Ainsi la zone hachurée n'est pas mesurable dans ces conditions instrumentales. La ligne rouge correspond à la bordure du continuum d'excitation électron-trou et donne donc une énergie caractéristique de la physique du système. Comme nous le verrons dans ce qui suit, cette bordure indique l'énergie minimale à fournir pour créer une excitation dans l'état supraconducteur. Sa forme est donc directement liée à la symétrie et à l'amplitude du gap supraconducteur, ainsi qu'à la topologie de la surface de Fermi. Son amplitude est maximale au vecteur d'onde $\mathrm{AF}$ et est d'environ $75 \mathrm{meV}$ au dopage optimal. La résolution instrumentale est donc suffisante pour mesurer des signaux confinés sous la bordure du continuum, tel que le pic de résonance magnétique apparaissant à environ $40 \mathrm{meV}$ au dopage optimal. Néanmoins, nous observons que la surface de l'ellipsoïde représente une portion non négligeable de l'espace physique. C'est essentiellement la résolution en vecteur d'onde qui est mauvaise par rapport à la physique. En énergie, la résolution est satisfaisante $(\sim 4 \mathrm{meV})$ par rapport à l'amplitude du continuum, $\left(\omega_{c} \sim 75 \mathrm{meV}\right)$.

Il est important de réaliser que l'image de la Fig. 9 n'est pas figée, dans le sens où on peut chercher des conditions expérimentales pour améliorer la résolution suivant une certaine direction, ce qui d'ailleurs fait partie de la richesse de la technique neutron. Par exemple, pour améliorer notre 


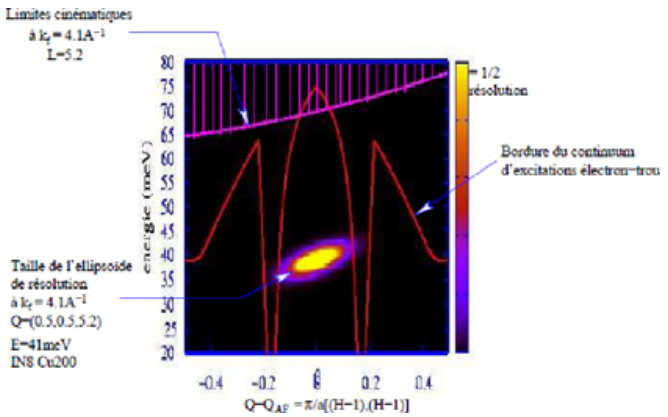

Figure 9. Taille de la résolution expérimentale $v s$ taille de la physique sur un spectrométre 3-axes thermiques (2T, IN8) [57] Sur cette figure est reporté l'ellipsoïde de résolution au point $\mathrm{AF}(0.5,0.5,5.2)$ à $39 \mathrm{meV}$ dans le plan $\left(\mathrm{Q}_{/ /(11)}, \omega\right)$. La ligne rouge est associée à la symétrie $\mathrm{d}_{x^{2}-y^{2}}$ du gap supraconducteur dans les cuprates (voir texte). La ligne violette indique, pour un vecteur d'onde donné, l'énergie de transfert maximale accessible. Pour les énergies supérieures, les conditions cinématiques ne sont plus satisfaites.

résolution en vecteur d'onde, on peut essayer d'insérer des collimateurs, au prix toutefois d'une certaine perte de flux. Le but est d'obtenir le meilleur compromis entre un flux important et une certaine qualité de résolution désirée par l'expérimentateur. Le niveau d'exigence et de précision des expériences sur les cuprates augmentant d'année en année, les expérimentateurs explorent de plus en plus les détails du spectre des fluctuations magnétiques. La prise en compte de la résolution instrumentale devient donc nécessaire dans l'analyse des résultats. Le but est alors d'extraire la physique intrinsèque du système, déconvoluée de la fonction de résolution. Pour cela, on postule une forme de la susceptibilité magnétique et on effectue numériquement le produit de convolution à 4 dimensions de cette forme avec la fonction de résolution (voir les cours de B. Hennion et de J. Ollivier).

\subsection{Une excitation magnétique apparaît dans l'état supraconducteur des cuprates : le pic de résonance magnétique}

Une des principales découvertes réalisées en utilisant la diffusion inélastique des neutrons est l'observation d'une excitation magnétique non conventionnelle dans l'état supraconducteur des cuprates : le pic de résonance magnétique. La première mesure de cette excitation fut réalisée par
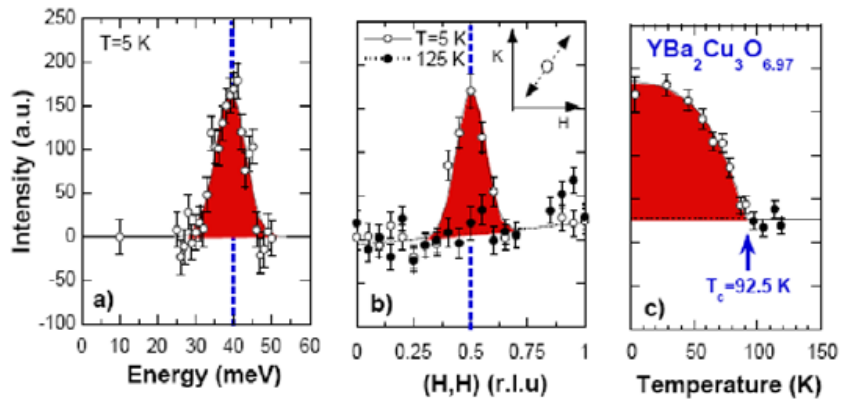

Figure 10. Mesures neutroniques du pic de résonance magnétique dans le composé $\mathrm{YBa}_{2} \mathrm{Cu}_{3} \mathrm{O}_{6.97}$ [8,62]. (gauche) Mesure en fonction de l'énergie et au vecteur d'onde $\vec{q}_{A F}=(0.5,0.5)$ à $5 \mathrm{~K}$ dans l'état supraconducteur. (milieu) Coupe en fonction du vecteur d'onde $\vec{q}$ suivant la direction (110) à une énergie constante de $40 \mathrm{meV}$ en dessous de $\mathrm{T}_{c}$ à $5 \mathrm{~K}$ (cercles ouverts) et au-dessus à $125 \mathrm{~K}$ (ronds noirs). Le pic apparaissant à basse température correspond au pic de résonance magnétique. (droite) Dépendance en température de son intensité enregistrée au vecteur $\vec{q}_{A F}$ et à $40 \mathrm{meV}$. 
J. Rossat-Mignot et al. en 1991 [62] dans un échantillon de $\mathrm{YBa}_{2} \mathrm{Cu}_{3} \mathrm{O}_{6.92}\left(\mathrm{~T}_{c}=91 \mathrm{~K}\right)$ puis, dans la même famille de cuprate, par de nombreux neutroniciens [8,25,26,50]. Comme le montre la Fig. 10, dans l'état supraconducteur, le pic de résonance magnétique se présente sous la forme d'une excitation étroite en énergie localisée à $E_{r} \sim 41 \mathrm{meV}$ et centrée sur le vecteur d'onde $\mathbf{Q}_{A F}=(0.5,0.5, \mathrm{~L})$ qui est caractéristique des corrélations AF dans les plans $\mathrm{CuO}_{2}$. En augmentant la température, l'énergie caractéristique du pic de résonance magnétique demeure inchangée alors que son intensité présente une dépendance en température similaire à celle d'un paramètre d'ordre, avec une disparition brutale à $\mathrm{T}_{c}$. Des mesures en neutrons polarisés sous champ ont permis de confirmer que ce pic de résonance est une excitation triplet de spin $(S=1)$. L'observation de ce pic magnétique a par la suite été reproduite pour d'autres familles d'oxydes de cuivre supraconducteurs. A dopage optimal, cette excitation est observée à $43 \mathrm{meV}$ dans le composé $\mathrm{Bi}_{2} \mathrm{Sr}_{2} \mathrm{CaCu}_{2} \mathrm{O}_{8+\delta}\left(\mathrm{T}_{c}=91 \mathrm{~K}\right)$ [23] et à $47 \mathrm{meV}$ dans le composé $\mathrm{Tl}_{2} \mathrm{Ba}_{2} \mathrm{CuO}_{4}$ $\left(\mathrm{T}_{c}=90 \mathrm{~K}\right)$ [30]. Pour les composés supraconducteurs dont la température critique à dopage optimal est de l'ordre de $90 \mathrm{~K}$, l'existence du pic de résonance semble une propriété générique.

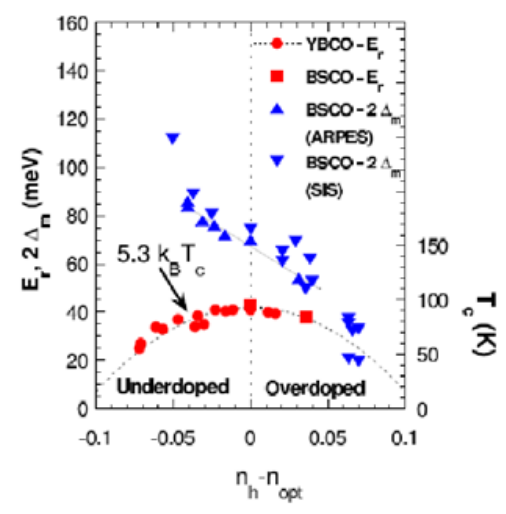

Figure 11. Energie du pic de résonance magnétique en fonction du dopage en trous [7]. La position en énergie du pic de résonance magnétique, $E_{r}$, observé dans les familles de cuprates $\mathrm{YBa}_{2} \mathrm{Cu}_{3} \mathrm{O}_{6+x}$ (ronds rouges) $[8,18,24,25,50,62]$ et $\mathrm{Bi}_{2} \mathrm{Sr}_{2} \mathrm{CaCu}_{2} \mathrm{O}_{8+\delta}$ (carrés rouges) [23,31], est représentée en fonction du dopage en trous. La ligne pointillée noire passant par l'ensemble des points correspond à $5.3 \mathrm{k}_{B} \mathrm{~T}_{c}$. Les points bleus triangulaires représentent la position en énergie de 2 fois le maximum du gap supraconducteur obtenu par ARPES [48] et tunneling [70].

Sur la Fig. 11, les positions en énergie des pic de résonance mesurés dans les familles de cuprates $\mathrm{YBa}_{2} \mathrm{Cu}_{3} \mathrm{O}_{6+x}[8,18,24,25,50,62]$ et $\mathrm{Bi}_{2} \mathrm{Sr}_{2} \mathrm{CaCu}_{2} \mathrm{O}_{8+\delta}$ [23,31] sont représentées en fonction du dopage en trous (pris par rapport au dopage à la $\mathrm{T}_{c}$ optimale). Les points reproduisent la forme du dôme supraconducteur indiquant que l'énergie du pic de résonance est proportionnelle à la $\mathrm{T}_{c}$ avec un rapport d'environ 5.3. Sur la même figure sont représentées les positions en énergie de 2 fois le maximum du gap supraconducteur, $2 \Delta_{0}$ mesuré par ARPES [48] et tunneling [70], en fonction du dopage. Comme nous le verrons plus en détail ci-dessous, cette quantité est une bonne approximation de l'énergie minimale à fournir au système pour faire une excitation élémentaire de l'état supraconducteur au vecteur d'onde AF. Autrement dit, en dessous de cette énergie, le système est dit «gappé ». On remarque alors que le pic de résonance magnétique se trouve toujours dans la région gappée du spectre.

Dans les composés à 2 plans $\mathrm{CuO}_{2}$ par maille élémentaire (systèmes biplans) tels que $\mathrm{YBa}_{2} \mathrm{Cu}_{3} \mathrm{O}_{6+x}$ et $\mathrm{Bi}_{2} \mathrm{Sr}_{2} \mathrm{CaCu}_{2} \mathrm{O}_{8+\delta}$, l'existence d'un couplage inter-plan (magnétique ou électronique) permet de lever la dégénérescence des signaux magnétiques associés à chaque plan. On distingue alors deux canaux qui, par référence aux modes d'ondes de spins dans l'état isolant, sont nommés canal acoustique (ac) et 


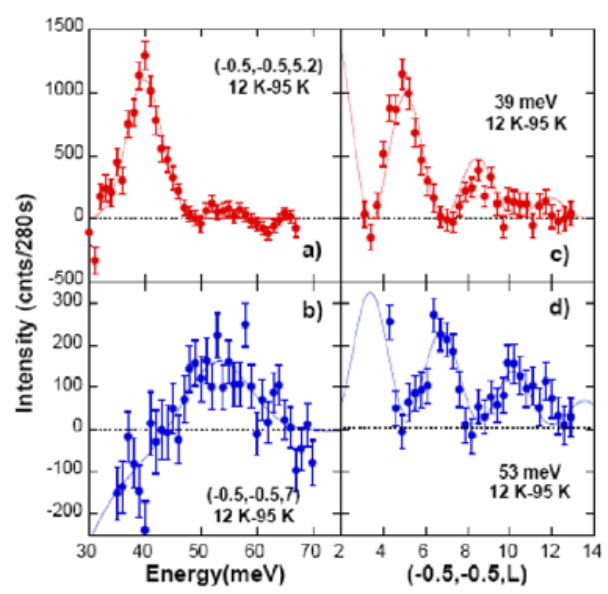

Figure 12. Mesures neutroniques des pics de résonances magnétiques acoustique et optique dans le composé $\mathrm{YBa}_{2} \mathrm{Cu}_{3} \mathrm{O}_{6.89}\left(\mathrm{~T}_{c}=89 \mathrm{~K}\right)$ [58]. Il est reporté sur les figures les différences $12 \mathrm{~K}\left(<\mathrm{T}_{c}\right)$ et $95 \mathrm{~K}\left(>\mathrm{T}_{c}\right)$ afin de mettre en évidence l'amplification de la réponse magnétique à travers $\mathrm{T}_{c}$. a) et c) Mesures du pic de résonance magnétique acoustique. La figure a) montre la différence $12 \mathrm{~K}-95 \mathrm{~K}$ des mesures en énergie à $\vec{q}_{A F}=(0.5,0.5)$ et à la composante suivant l'axe c de $L=5.2$ correspondant à un maximum du facteur de structure acoustique, Eq. (2.5). Le pic de résonance acoustique apparaît à $40 \mathrm{meV}$. La figure b) correspond à la différence $12 \mathrm{~K}-95 \mathrm{~K}$ des coupes au vecteur planaire $\vec{q}_{A F}$ et à une énergie de $40 \mathrm{meV}$ en fonction de la composante $L$. La ligne rouge correspond à la modulation sinusoïdale de l'Eq. (2.5) multipliée par le facteur de forme magnétique (enveloppe du signal). b et d Mesures du pic de résonance magnétique optique. Le pic de résonance optique apparaît à $53 \mathrm{meV}$ (a) et a une modulation en cosinus (d) suivant l'axe c donné par l'Eq. (2.5).

optique (op). La section efficace neutron s'écrit alors :

$$
\operatorname{Im} \chi(\vec{Q}, \omega)=\sin ^{2}\left(\pi \frac{d \cdot L}{c}\right) \operatorname{Im} \chi_{a c}(\vec{q}, \omega)+\cos ^{2}\left(\pi \frac{d \cdot L}{c}\right) \operatorname{Im} \chi_{o p}(\vec{q}, \omega)
$$

où $\mathrm{d} \sim 3,3 \AA$ et $\mathrm{c} \sim 11.7 \AA$ représentent respectivement la distance inter-plan et le paramètre de maille suivant l'axe c, $\vec{q}=(H, K)$ correspond à la composante planaire du vecteur $\vec{Q}$ et L la composante suivant l'axe $\vec{c}$.

Dans ces systèmes biplans, deux pics de résonances magnétiques d'énergies différentes et, étrangement, d'intensités différentes sont observés [58,59]. Ainsi dans le composé $\mathrm{YBa}_{2} \mathrm{Cu}_{3} \mathrm{O}_{6.89}$ $\left(\mathrm{T}_{c}=89 \mathrm{~K}\right)$, le pic de résonance acoustique apparaît à $41 \mathrm{meV}$ alors que le pic de résonance optique est observé à $53 \mathrm{meV}$, avec une intensité 6 fois plus petite. La différence d'intensité des pics est une propriété remarquable qui ne peut pas être expliquée par la seule levée de dégénérescence induite par exemple par un couplage AF entre plans.

\subsection{Dispersion du pic de résonance magnétique : un spectre magnétique en forme de « $\mathrm{X}$ »}

Les pics de résonances magnétiques (acoustique et optique) sont observés dans l'état supraconducteur au vecteur d'onde AF, mais l'observation ne se limite pas là. En effet, il a été montré que le pic de résonance magnétique fait en réalité partie de tout un "mode magnétique résonant" dont l'énergie dépend du vecteur d'onde $[4,9,17,18,22,32,58]$. La Fig. 13 illustre ce mode magnétique mesuré dans un échantillon $\mathrm{YBa}_{2} \mathrm{Cu}_{3} \mathrm{O}_{6.85}$ dans lequel le pic de résonance magnétique est observé à 40 meV. En se déplaçant de la position en énergie du pic de résonance, à 35 et $38 \mathrm{meV}$ par exemple (Fig. 13 de gauche), des pics magnétiques similaires au pic de résonance apparaissent dans l'état supraconducteur de part et d'autre du vecteur d'onde AF, suivant les directions $a^{*}(100)$ et b*(010). 

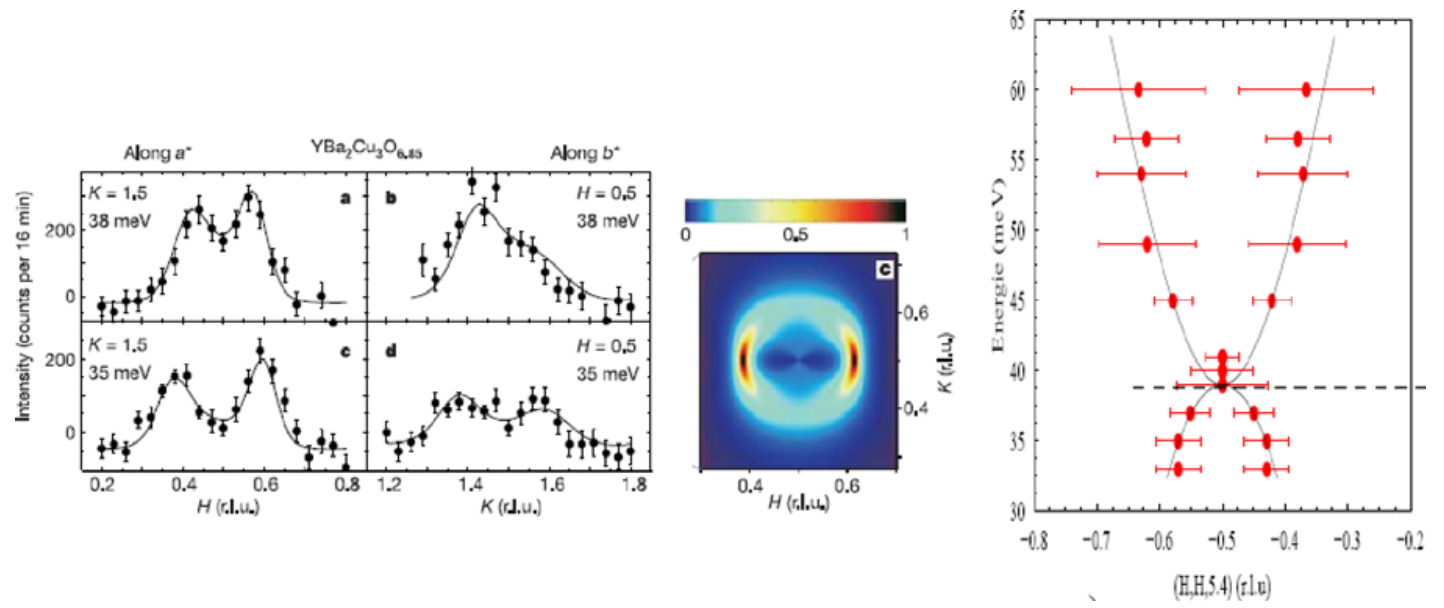

Figure 13. Dispersion du pic de résonance magnétique acoustique mesurée dans $\mathrm{YBa}_{2} \mathrm{Cu}_{3} \mathrm{O}_{6.85}\left(\mathrm{~T}_{c}=89 \mathrm{~K}\right.$, $E_{r}=40 \mathrm{meV}$ ) $[9,33,58]$. (gauche) Différences $10 \mathrm{~K}-100 \mathrm{~K}$ des coupes à énergie constante suivant les directions $\mathrm{a}^{*}(100)$ et $\mathrm{b}^{*}(010)$ à 38 et $35 \mathrm{meV}$. De façon similaire au pic de résonance magnétique à $\vec{q}_{A F}$, des pics magnétiques apparaissent dans l'état supraconducteur sur des positions incommensurables $\vec{q}_{A F}+\vec{\delta}$. Notons que la différence d'intensité entre deux pics d'une même coupe s'interprète par des effets de convolution de la résolution instrumentale avec le signal physique (voir cours B. Hennion). En revanche, les différences d'intensités des pics suivant les axes $\mathrm{a}^{*}$ et $\mathrm{b}^{*}$ sont réelles et indiquent que la distribution d'intensité magnétique n'est pas isotrope dans la plan de base. (milieu) Cartographie de l'intensité magnétique dans le plan de base à une énergie de $35 \mathrm{meV}$ après soustraction de la résolution instrumentale. La carte représente donc le signal physique intrinsèque. La convolution de ce signal par la fonction de résolution correspond aux lignes noires de la figure de gauche. (droite) Positions des pics magnétiques obtenues par des ajustements gaussiens des différences $10 \mathrm{~K}-100 \mathrm{~K}$ des coupes à énergie constante. C'est en fait tout un spectre magnétique en forme de «X»qui apparaît dans l'état supraconducteur. Le centre du X à $\vec{q}_{A F}$ et $\mathrm{E}_{r}=40 \mathrm{meV}$ correspond au pic de résonance observé par J. Rossat-Mignot et al. en 1991 [62]).

L'intensité magnétique a été cartographiée dans le plan de base pour une énergie de $35 \mathrm{meV}$ (Fig. 13 milieu). L'intensité magnétique est distribuée sur un cercle, avec des maxima suivant l'axe a*. Enfin, sur la Fig. 13 de droite sont reportées les positions en vecteur d'onde des pics magnétiques suivant la direction (110) en fonction de l'énergie. C'est en fait tout un mode magnétique global en forme de sablier ou de $X$ contenu dans une fenêtre en énergie allant de $33 \mathrm{meV}$ à $65 \mathrm{meV}$ qui apparât dans l'état supraconducteur. Le centre de ce mode à $\vec{q}_{A F}$ et $\mathrm{E}_{r}=40 \mathrm{meV}$ correspond au pic de résonance magnétique.

\section{INTERPRÉTATION DES EXCITATIONS MAGNÉTIQUES RÉSONNANTES DANS UNE DESCRIPTION ITINÉRANTE : LE MODÈLE DE L'EXCITON DE SPIN}

Les neutrons mettent donc en évidence l'apparition d'un mode magnétique dans l'état supraconducteur, avec une structure dans l'espace $(\vec{Q}, \omega)$ en forme de sablier (ou de «X») centré au vecteur d'onde antiferromagnétique. Toute description microscopique du système doit pouvoir reproduire une susceptibilité magnétique générant ce « $\mathrm{X} »$ magnétique dans l'état supraconducteur. Dans cette partie, nous donnons une description de la susceptibilité de spin généralisée sur la base d'un modèle itinérant. Nous montrons comment, dans cette approche, les principales caractéristiques du spectre résonant magnétique en forme de « $\mathrm{X}$ » peuvent être interprétées comme un mode d'exciton de spin confiné dans un espace «gappé» ou vide d'excitations élémentaires de type électron-trou. 


\subsection{Structure électronique mesurée par photoémission résolue en angle}

Comme nous le verrons ci-dessous, il est essentiel d'avoir une idée précise de la structure électronique $\epsilon_{\vec{k}}$ pour calculer la susceptibilité magnétique. Pour cela, au cours de ces 20 dernières années, les cuprates ont été un des moteurs principaux du développement de la technique de photoémission résolue en angle (ou Angle Resolved PhotoEmission Spectroscopy, ARPES, en anglais). Le lecteur trouvera tous les détails de la technique et de ses applications à l'étude des cuprates dans les références suivantes $[11,19,34]$. L'ARPES consiste à illuminer l'échantillon par un rayonnement UV (typiquement entre $10 \mathrm{eV}$ et $100 \mathrm{eV}$ ) et à analyser les directions et énergies des électrons photoémis. Sous certaines approximations, l'intensité ARPES I $(\vec{k}, \omega)$ est directement proportionnelle à la fonction spectrale à 1 particule $\mathrm{A}(\vec{k}, \omega)$. $\mathrm{A}(\vec{k}, \omega)$ est la représentation spectrale (ou de Lehman) de la fonction de Green [1]. $\mathrm{A}(\vec{k}, \omega)$ représente donc la probabilité d'extraire ou d'ajouter un électron au système en interaction ${ }^{1}$. La technique ARPES permet de cartographier en énergie et en vecteur d'onde (ainsi qu'en température) cette fonction. Ainsi :

$$
A(\vec{k}, \omega)=\frac{1}{\pi} \operatorname{Im} G\left(\vec{k}, \omega+i 0^{+}\right)
$$

La fonction de Green à 1 particule, $\mathrm{G}(\vec{k}, \omega)$, peut-être exprimée en fonction de la fonction de Green à 1 particule sans interaction, $G^{0}(\vec{k}, \omega)=1 /\left[\omega-\epsilon_{\vec{k}}^{0}\right]$, où $\epsilon_{\vec{k}}^{0}$ est l'énergie des quasiparticules sans interaction, et de la self énergie $\Sigma(\vec{k}, \omega)$ :

$$
G(\vec{k}, \omega)^{-1}=G^{0}(\vec{k}, \omega)^{-1}-\Sigma(\vec{k}, \omega)
$$

Expérimentalement, dans les cuprates, on observe une faible dépendance en vecteur d'onde de la self énergie et notamment de sa partie imaginaire au voisinage du niveau de Fermi [36,60]. Il est donc généralement supposé que $\Sigma(\vec{k}, \omega)=\Sigma(\omega)$. L'expression de la fonction spectrale à 1 électron en fonction des composantes réelle, $\Sigma^{\prime}$, et imaginaire, $\Sigma^{\prime \prime}$, de la self énergie est :

$$
A(\vec{k}, \omega)=\frac{1}{\pi} \frac{\Sigma^{\prime \prime}(\omega)}{\left[\omega-\xi_{\vec{k}}^{0}-\Sigma^{\prime}(\omega)\right]^{2}-\Sigma^{\prime \prime}(\omega)}
$$

où $\xi_{\vec{k}}^{0}=\epsilon_{\vec{k}}^{0}-\mu, \mu$ étant le potentiel chimique. $\Sigma^{\prime}$ et $\Sigma^{\prime \prime}$ sont reliées par une transformation de KramersKronig (KK).

A une énergie donnée, $\mathrm{A}(\vec{k})$ est une lorentzienne dont les pôles apparaissent aux vecteurs d'ondes $\vec{k}_{m}(\omega)$ satisfaisant la condition : $\omega-\xi_{\vec{k}_{m}}=0$, où $\xi_{\vec{k}_{m}}=\xi_{\vec{k}_{m}}^{0}-\Sigma^{\prime}(\omega)$ correspond à la dispersion des quasiparticules «habillées » par les interactions. C'est cette dispersion qui est directement sondée par ARPES. Sur la Fig. 14 de gauche, nous reportons une cartographie $\left(\mathrm{k}_{x}, \mathrm{k}_{y}\right)$ de l'intensité ARPES obtenue au niveau de Fermi dans le cuprate $(\mathrm{Bi}, \mathrm{Pb})_{2} \mathrm{Sr}_{2} \mathrm{CaCu}_{2} \mathrm{O}_{8+\delta}$ [40]. La surface de Fermi dessinée par les lignes lumineuses est ouverte en bord de zone au point $M$ et formée de poches de trous centrées au point $Y$. La figure de droite schématise la nappe de dispersion des quasiparticules en indiquant la région des états occupés en dessous de $\mathrm{E}_{F}$. Il est également possible d'étudier la dispersion des quasiparticules suivant une certaine direction dans l'espace réciproque. Ainsi, nous montrons sur la Fig. 14 (centre) la cartographie $(\vec{k}, \omega)$ de la dispersion $\epsilon_{\vec{k}}$ suivant une direction $\vec{k} / /(\Gamma-\mathrm{Y})$. En linéarisant la relation de dispersion au niveau de Fermi, on obtient : $\Sigma^{\prime}(\omega)=\omega-v_{F}^{0}\left(k_{m}(\omega)-k_{F}\right)$, où $k_{F}=k_{m}\left(\omega=E_{f}\right)$ et $v_{F}^{0}$ est la vitesse de Fermi sans interaction. D'autre part, la largeur expérimentale en vecteur d'onde de la lorentzienne, notée $\mathrm{W}(\omega)$, est directement proportionnelle à la partie imaginaire

\footnotetext{
${ }^{1} \mathrm{~A}(\vec{k}, \omega)$ peut être décrite par la somme de deux termes : $\mathrm{A}(\vec{k}, \omega)=\mathrm{A}_{-}(\vec{k}, \omega)+\mathrm{A}_{+}(\vec{k}, \omega)$. Si $|m\rangle$ et $|n\rangle$ sont des états propres du système avec des énergies $\epsilon_{n}$ et $\epsilon_{m}, \mathrm{Z}$ la fonction de partition, alors : $\mathrm{A}_{-}(\vec{k}, \omega)=Z^{-1} \sum_{m, n} \exp -\beta \epsilon_{m}\left|\left\langle n\left|c_{\vec{k}}^{\dagger}\right| m\right\rangle\right|^{2} \delta\left(\omega+\epsilon_{m}-\epsilon_{n}\right)$ correspond à la probabilité d'extraire un électron et $\mathrm{A}_{+}(\vec{k}, \omega)=Z^{-1} \sum_{m, n} \exp -\beta \epsilon_{m}\left|\left\langle n\left|c_{\vec{k}}\right| m\right\rangle\right|^{2} \delta\left(\omega+\epsilon_{n}-\epsilon_{m}\right)$ à celle d'ajouter un électron. $\mathrm{A}_{+}$et $\mathrm{A}_{-}$sont reliées à $\mathrm{A}(\vec{k}, \omega)$ par la fonction de Fermi, $f(\omega): \mathrm{A}_{+}=[1-f(\omega)] A(\vec{k}, \omega)$ et $\mathrm{A}_{-}=f(\omega) A(\vec{k}, \omega)$. L'intensité ARPES est proportionnelle à A_.
} 

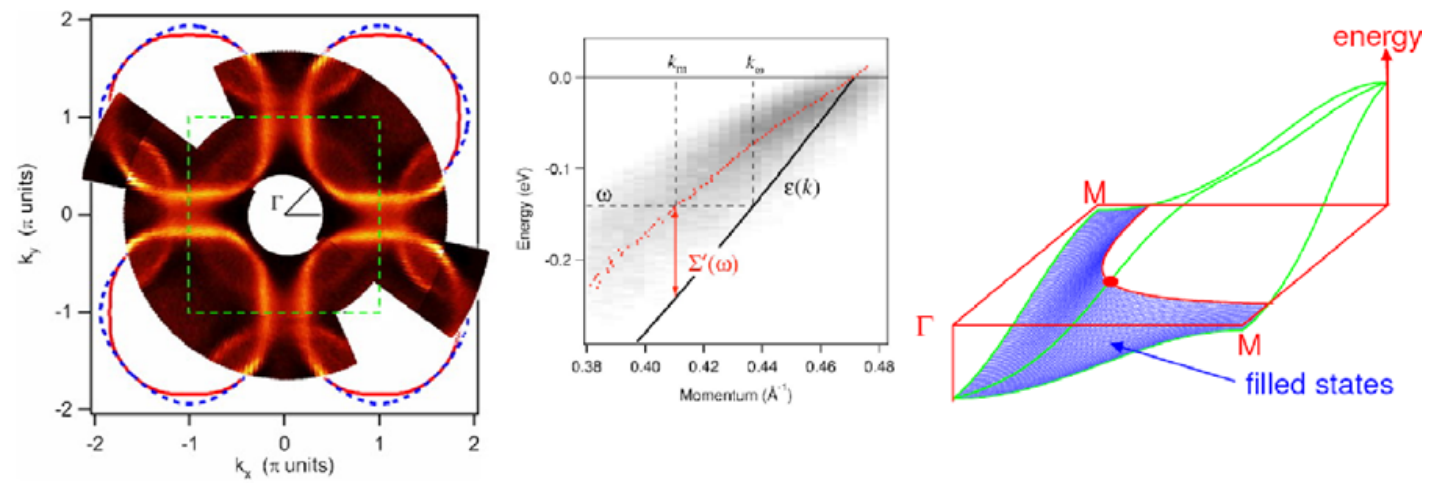

Figure 14. Mesure ARPES dans le cuprate $(\mathrm{Bi}, \mathrm{Pb})_{2} \mathrm{Sr}_{2} \mathrm{CaCu}_{2} \mathrm{O}_{8+\delta}$ [40,41]. (gauche) Cartographie $\left(k_{x}, k_{y}\right)$ de la surface de Fermi. Le carré pointillé vert délimite la $1^{r e}$ zone de Brillouin. Les lignes rouges correspondent à un ajustement par un modèle de liaisons fortes et les lignes bleues correspondent aux prédictions des calculs LDA. (milieu) cartographie $(\vec{k}, \omega)$ de la dispersion suivant une direction $\vec{k} / /(\Gamma-Y)$. Les points rouges indiquent la position du maximum d'intensité de la dispersion. La ligne noire indique la position de la dispersion sans interaction $\epsilon_{\vec{k}}^{0}$. Comme indiqué par la double flèche rouge, les différences des positions en énergie des dispersions avec et sans interaction sont données par $\Sigma^{\prime}(\omega)$. (droite) Schéma de la nappe de dispersion des états de quasiparticules dans les cuprates. La région des états occupés est coloriée en bleu.

de la self énergie : $\Sigma^{\prime \prime}(\omega)=-v_{F}^{0} W(\omega)$. En analysant les données de manière self-consistente via la transformation de Kramers-Kronig, il est possible d'avoir accés à $v_{F}^{0}\left(i . e . \xi^{0}(\vec{k})\right), \Sigma^{\prime \prime}(\omega)$ et $\Sigma^{\prime}(\omega)$ [47,53].

Il est utile de rechercher un fit de la nappe de dispersion, $\xi_{\vec{k}}$, mesurée par ARPES. Un modèle de type liaisons fortes est généralement utilisé avec un certain nombre de paramètres ajustables : $t_{0}, t_{1}, t_{2} \ldots$ Ces paramètres s'interprètent comme des intégrales de sauts entre $1^{e r}, 2^{m e}, 3^{m e}$, etc voisins.

$$
\begin{aligned}
\xi_{\vec{k}}= & t_{0}+t_{1} \frac{\cos \left(k_{x}\right)+\cos \left(k_{y}\right)}{2}+t_{2} \cos \left(k_{x}\right) \cos \left(k_{y}\right)+t_{3} \frac{\cos \left(2 k_{x}\right)+\cos \left(2 k_{y}\right)}{2} \\
& +t_{4} \frac{\cos \left(2 k_{x}\right) \cos \left(k_{y}\right)+\cos \left(k_{x}\right) \cos \left(2 k_{y}\right)}{2}+t_{5} \cos \left(2 k_{x}\right) \cos \left(2 k_{y}\right)
\end{aligned}
$$

Les rapports des paramètres $t_{i}(i=1-5) / \mathrm{t}_{0}$ sont fixés par le fit de la surface de Fermi. La dispersion en énergie permet d'obtenir $\mathrm{t}_{0}$.

\subsection{Susceptibilité magnétique sans interaction dans l'état normal}

Nous considérons tout d'abord un métal sans interaction où encore un «bon liquide de Fermi ». Celuici est donc constitué de quasiparticules cohérentes ayant des propriétés proches de l'électron et une relation de dispersion $\varepsilon_{k, \sigma}^{0}$ que l'on supposera indépendante de l'état de spin: $\varepsilon_{k, \sigma}=\varepsilon_{k}$. Une excitation magnétique implique le transfert d'un état occupé $|\mathrm{k}, \sigma\rangle$ à un état inoccupé $|\mathrm{k}+\mathrm{q},-\sigma\rangle$. C'est ce que l'on nomme excitation de type électron-trou (e-t). Ce type d'excitation coûte une énergie $\varepsilon_{\vec{k}+\vec{q}}^{0}-\varepsilon_{\vec{k}}^{0}$. La susceptibilité sans interaction, $\chi_{0}$, qui décrit ces excitations $(e-t)$ avec renversement du spin est donnée par la fonction de Lindhardt [44] :

$$
\chi_{0}(\vec{q}, \omega)=-\frac{1}{\left(g \mu_{B}\right)^{2}} \lim _{\delta \rightarrow 0} \sum_{\vec{k}} \frac{f_{\vec{k}+\vec{q}}-f_{\vec{k}}}{\xi_{\vec{k}+\vec{q}}^{0}-\xi_{\vec{k}}^{0}-\hbar \omega-i \delta}
$$

où $\xi_{\vec{k}}^{0}$ est définie à partir de la relation de dispersion des quasiparticules $\epsilon_{k}^{0}$ et du potentiel chimique $\mu$ : $\xi_{\vec{k}}^{0}=\epsilon_{k}^{0}-\mu . f_{\vec{k}}$ correspond à la distribution de Fermi. En terme de fonction de Green, $\chi_{0}(\vec{q}, \omega)$ apparait 
comme une fonction d'autocorrélation :

$$
\chi_{0}(\vec{q}, \omega) \propto-2 i \int G(\vec{k}, \omega) G(\vec{k}+\vec{q}, \omega+\Omega) \delta \vec{k} \delta \Omega
$$

La relation de dispersion des quasiparticules et le potentiel chimique contrôlent à eux seuls $\chi_{0}$. Des effets tels que la proximité d'une singularité de Van Hove ou des propriétés d'emboîtement (nesting) statique ou dynamique donnent les principales contributions à $\chi_{0}$. Ce sont essentiellement les processus à $\mathbf{q}=\mathbf{2} \mathbf{k}_{\mathbf{F}}$, où $k_{F}$ est un vecteur d'onde d'une quasiparticule au niveau de Fermi, qui contribuent à la susceptibilité sans interaction.

Dans le modèle de l'exciton de spin, l'objectif est de modéliser les modifications du spectre magnétique engendrées par l'apparition du gap supraconducteur de symétrie $d$. Ainsi, l'état microscopique virtuel du système dit «sans interaction»est pris comme étant l'état normal physique non supraconducteur où le spectre des fluctuations magnétiques se limite à un continuum non structuré d'excitations (el-tr). Comme nous l'avons montré dans le chapitre introductif, si cette approximation est justifiée dans le régime sur-dopé, elle est beaucoup moins réaliste dans le régime sous-dopé. La fonction de Lindhardt (Eq. (3.5)) a ainsi été étudiée en fonction de la topologie de la surface de Fermi mesurée par ARPES dans l'état normal [51,55,55]. Nous montrons sur la Fig. 15 un exemple de calcul de la partie réelle de la susceptibilité de Lindhardt [51]. Les lignes intenses qui apparaissent en des positions incommensurables correspondent aux processus à $2 \mathrm{k}_{F}$ passant par le centre de zone $\Gamma$. En effet, du fait de la symétrie, ce type de processus a une multiplicité de 8. La structure autour de point $Y$ dépend fortement de la position du point «selle» de la nappe de dispersion par rapport à l'énergie de Fermi.
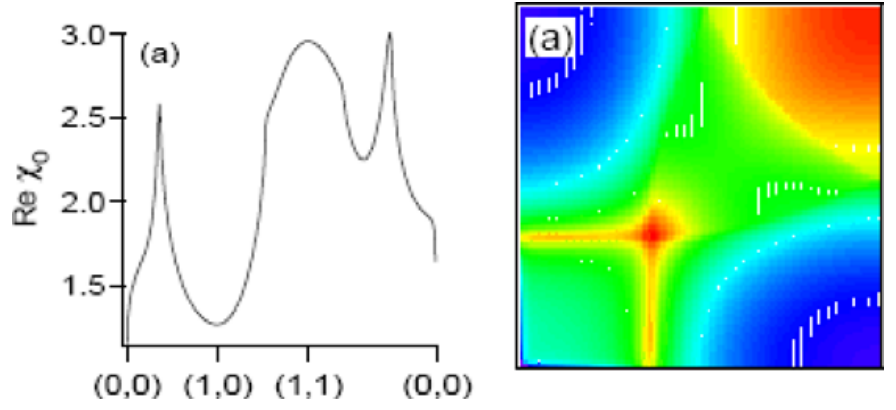

Figure 15. Calcul de la partie réelle de la susceptibilité de Lindhardt à partir de la mesure ARPES dans le cuprate $\mathrm{Bi}_{2} \mathrm{Sr}_{2} \mathrm{CaCu}_{2} \mathrm{O}_{8+\delta}$ [51]. (gauche) Calcul de $\operatorname{Re} \chi_{0}(q, \omega=0)$ dans les différentes directions de symétrie de la zone de Brillouin. (droite) Cartographie $\left(k_{x}, k_{y}\right)$ de $\operatorname{Re} \chi_{0}(q, \omega=0)$.

\subsection{Susceptibilité sans interaction dans l'état supraconducteur, continuum d'excitations électrons-trous et termes d'interférences}

Dans un supraconducteur, la susceptibilité sans interaction est modifiée du fait de l'appariement des porteurs de charge en paires de Cooper. En entrant dans la phase supraconductrice, un gap s'ouvre dans le spectre des excitations de basses énergies en suivant la symétrie du paramètre d'ordre. La relation de dispersion des quasiparticules excitées dans l'état supraconducteur devient :

$$
E_{\vec{k}}=\sqrt{\Delta_{\vec{k}}^{2}+\xi_{\vec{k}}^{2}}
$$


La symétrie du paramètre d'ordre supraconducteur dans les suprates est, en première approximation, de type $\mathrm{d}_{x^{2}-y^{2}}$ :

$$
\Delta(\vec{k})=\Delta_{0}\left(\cos \left(k_{x} a\right)-\cos \left(k_{y} a\right)\right) / 2
$$

Ajoutons que des études ARPES montrent quelques déviations par rapport à la symétrie $d$ [48,54].

La susceptibilité BCS dans l'état supraconducteur est alors donnée par [51,56] :

$$
\begin{array}{rl}
\chi_{0}{ }^{B C S}(\vec{q}, \omega)=-\left(g \mu_{B}\right)^{2} \lim _{\epsilon \rightarrow 0} & 2 \sum_{\vec{k}}\left[1-\frac{\Delta_{\vec{k}} \Delta_{\vec{k}+\vec{q}}+\xi_{\vec{k}+\vec{q}} \xi_{\vec{k}}}{E_{\vec{k}+\vec{q}} E_{\vec{k}}}\right] \frac{1}{4} \frac{1-f_{\vec{k}+\vec{q}}-f_{\vec{k}}}{E_{\vec{k}+\vec{q}}+E_{\vec{k}}-\hbar \omega-i \epsilon} \\
+ & {\left[1+\frac{\Delta_{\vec{k}} \Delta_{\vec{k}+\vec{q}}+\xi_{\vec{k}+\vec{q}} \xi_{\vec{k}}}{E_{\vec{k}+\vec{q}} E_{\vec{k}}}\right] \frac{1}{2} \frac{f_{\vec{k}}-f_{\vec{k}+\vec{q}}}{\omega-E_{\vec{k}+\vec{q}}+E_{\vec{k}}+i \epsilon}}
\end{array}
$$

(Dans la limite $\Delta_{\vec{k}}$ tend vers zéro, l'Eq. (3.10) redonne l'Eq. (3.5).)
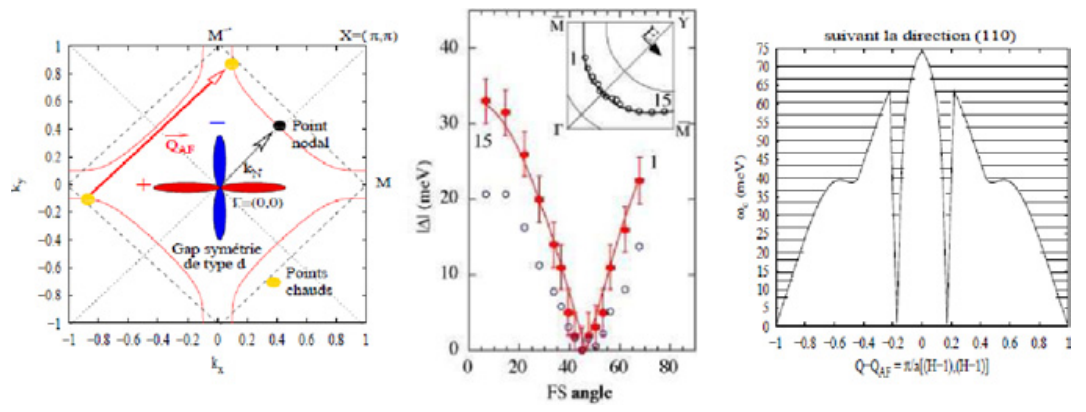

Figure 16. Bordure du continuum d'excitations el-t, $\omega_{c}(\vec{q})$, dans le cuprate $\mathrm{Bi}_{2} \mathrm{Sr}_{2} \mathrm{CaCu}_{2} \mathrm{O}_{8+\delta}$ [51]. (gauche) Résultat d'un ajustement utilisant un modèle de type liaison forte de la mesure ARPES. On indique sur la figure les points chauds de la surface de Fermi connectés par le vecteur d'onde AF ainsi que le vecteur $\mathrm{k}_{N}$ qui connecte le point $\Gamma$ au point nodal de la surface de Fermi ou l'amplitude du gap supraconducteur est nulle. (centre) Dépendance angulaire sur la surface de Fermi de la position en énergie du gap supraconducteur mesuré par ARPES. L'énergie du gap varie en $\mathrm{d}_{x^{2}-y^{2}}$ en s'annulant au point nodal à $45^{\circ}$ de la direction $\Gamma-M$. (droite) Calcul de la bordure du continuum, $\omega_{c}(\vec{q})$, à partir des données ARPES des figures de droite et du centre. Les zones hachurées indiquent la position du continuum d'excitations $e-t$ et, par conséquent, les zones blanches correspondent aux régions «gappées» du spectre (vide d'excitation $e-t)$.

Les susceptibilités magnétiques dans l'état normal et supraconducteur présentent deux différences principales. Tout d'abord, pour un vecteur $\vec{q}$, il est nécessaire de fournir une quantité d'énergie minimale, $\omega_{c}(\vec{q})$, pour pouvoir créer une excitation élémentaire de type $e$-t. Cette énergie dépend des valeurs du gap supraconducteur aux points de la surface de Fermi connectés par le vecteur d'onde $\vec{q}$ :

$$
\omega_{c}(\vec{q})=\operatorname{Min}\left(E_{\vec{k}+\vec{q}}+E_{\vec{k}}\right)
$$

$\omega_{c}(\vec{q})$ définit ce que l'on nomme la bordure du continuum d'excitations $e$ - $t$. Pour des énergies inférieures à $\omega_{c}(\vec{q})$, le spectre en $(\vec{q}, \omega)$ de la susceptibilité magnétique est vide de toutes excitations $e$ - $t$, il est dit « gappé ». La forme de $\omega_{c}(\vec{q})$ est fonction de la topologie de la surface de Fermi et de l'amplitude et de la symétrie du gap supraconducteur. Sur la Fig. 16 de droite, nous reportons le calcul de $\omega_{c}(\vec{q})$ autour du vecteur AF suivant la direction $\Gamma-M(110)$. Pour le calcul, nous avons utilisé l'ajustement par un modèle de liaisons fortes de la nappe de dispersion obtenue par ARPES dans $\mathrm{Bi}_{2} \mathrm{Sr}_{2} \mathrm{CaCu}_{2} \mathrm{O}_{8+\delta}$ [51] (fig. de gauche). Sur la figure du centre, nous montrons le gap supraconducteur de symétrie $\mathrm{d}_{x^{2}-y^{2}}$ avec $2 \Delta_{0}=80 \mathrm{meV}$ mesuré par ARPES [48] au voisinage du dopage optimal. Suivant la direction (110), $\omega_{c}(\vec{q})(\mathrm{q}=\mathrm{h}(1,1))$ tombe à énergie nulle aux vecteurs d'ondes joignant les points nodaux de la surface 
de Fermi où l'amplitude du gap SC est nulle : $2 k_{N}=(0.5 \pm \delta, 0.5 \pm \delta)$. Nous indiquons le vecteur $k_{N}$ sur la figure de gauche. Ces branches du continuum allant à énergie nulle ont été baptisées «bandes muettes » [58].

En théorie, les neutrons offrent l'unique possibilité instrumentale de mesurer la forme du continuum d'excitations $e$ - $t$. En pratique, l'intensité des excitations élémentaires du continuum est à la limite du seuil de détection actuel des neutrons sur 3-axes $\left(\sim 30 \mu_{B}^{2} \cdot \mathrm{eV}^{-1}\right)$. En revanche, nous allons montrer que le continuum pourrait être à l'origine de la structure dispersive particulière des signaux magnétiques mesurés dans l'état supraconducteur.

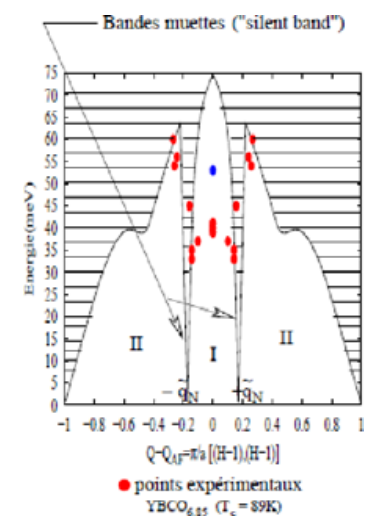

Figure 17. Les modes résonants magnétiques sous le continuum d'excitations $e-t$. Les points rouges correspondent aux positions des modes magnétiques apparaissant dans l'état supraconducteur Fig. 14. La ligne noire correspond à la bordure du continuum d'excitations el-t tracée sur la Fig. 16.

En effet, sur la Fig. 17, nous superposons les positions en énergie suivant la direction (110) du mode magnétique en forme de «X» observées par neutrons dans l'état supraconducteur (voir Fig. 14) et $\omega_{c}(\vec{q})$. Nous observons que les points expérimentaux sont confinés dans les régions « gappées ». Il est également surprenant de voir que l'interruption de la dispersion vers les basses énergies $\mathrm{du}$ « $\mathrm{X}$ » coïncide avec la position des bandes muettes. D'autre part, on observe une anomalie dans l'intensité de la branche de «X»dispersant vers les hautes énergies juste au croisement avec les bandes muettes [58]. Cette figure montre que les modes résonants peuvent être interprétés comme des excitations apparaissant sous le gap et dont la structure dispersive semble être guidée par la forme du continuum d'excitation $e$ - $t$. Les excitations $e-t$ du continuum pourraient être responsables d'un fort amortissement à l'origine de la suppression des modes magnétiques dans les zones non «gappées ». Ainsi, la forme du spectre magnétique serait imposée par celle de $\omega_{c}(\vec{q})$.

Les préfacteurs $1 \pm\left(\Delta_{\vec{k}} \Delta_{\vec{k}+\vec{q}}+\varepsilon_{\vec{k}+\vec{q}} \varepsilon_{\vec{k}} /\left(E_{\vec{k}+\vec{q}} E_{\vec{k}}\right)\right)$, appelés facteurs de cohérence, dépendent quant à eux de la phase du paramètre d'ordre supraconducteur. Ils correspondent respectivement à la création ou l'annihilation de paires d'excitations obtenues en brisant ou formant une paire de Cooper, et à la diffusion de quasiparticules. Ils contiennent des termes d'interférences de type $\Delta_{\vec{k}} \Delta_{\vec{k}+\vec{q}}$ provenant de la nature à deux particules des excitations magnétiques. Ces produits sondent le changement de signe du paramètre d'ordre supraconducteur. On remarque notamment que les maxima sont obtenus en satisfaisant la condition $\Delta_{\vec{k}} \Delta_{\vec{k}+\vec{q}}<0$ qui, dans les cuprates, est automatiquement satisfaite autour du vecteur d'onde planaire AF. L'existence d'une excitation AF telle que le pic de résonance magnétique dans l'état supraconducteur est donc en accord avec un gap supraconducteur de symétrie $\mathrm{d}_{x^{2}-y^{2}}$ maximisant le facteur de cohérence au vecteur d'onde AF. Il serait en revanche plus délicat de mesurer une excitation similaire à $\mathrm{q}=0$ où le facteur de cohérence est nul. De même, avec une symétrie isotrope $s$ du paramètre d'ordre, $\Delta_{\vec{k}}=\Delta_{s}$, il serait impossible d'observer un pic de résonance magnétique à $\mathrm{q}_{A F}$. 
Ainsi, la mesure de la susceptibilité magnétique dynamique par diffusion des neutrons permet de façon indirecte de déterminer la symétrie du paramètre d'ordre supraconducteur [45].

\subsection{Susceptibilité magnétique avec interaction dans le cadre de l'approximation de la Random Phase Approximation (RPA)}

Comme nous venons de le montrer, la structure dispersive particulière du mode magnétique résonant observé par neutrons dans l'état supraconducteur est reliée à la forme de la bordure du continuum d'excitations el-t. Pour rendre compte de ces modes magnétiques, nous considérons une amplification de la susceptibilité magnétique par les interactions traitées dans l'approximation RPA. En effet, la susceptibilité magnétique s'écrit alors en fonction de $\chi_{0}$ et d'un terme d'interaction (que nous allons appeler $I(\vec{q}))[51,55,56]$ :

$$
\chi(\vec{q}, \omega)_{R P A}=\frac{\chi_{0}(\vec{q}, \omega)}{1-V(\vec{q}) \chi_{0}(\vec{q}, \omega)}
$$

et donc,

$$
\operatorname{Im} \chi(\vec{q}, \omega)_{R P A}=\frac{\operatorname{Im} \chi_{0}(\vec{q}, \omega)}{\left(1-V(\vec{q}) \chi_{0}^{\prime}(\vec{q}, \omega)\right)^{2}+\left(V(\vec{q}) \operatorname{Im} \chi_{0}(\vec{q}, \omega)\right)^{2}}
$$

où $V(\vec{q})=\frac{2 I(\vec{q})}{\left(g \mu_{B}\right)^{2}}$. La susceptibilité RPA est une forme générale que l'on trouve dans les modèles de couplages forts et faibles. Les différences concernent l'expression de la susceptibilité dans l'état normal et la forme de l'interaction qui peut-être par exemple un $U$ dans le modèle de Hubbard à 1 seule bande ou encore $-J(\vec{q})=2 J\left(\cos \left(q_{x}\right)+\cos \left(q_{y}\right)\right)$ dans le modèle $t$ - $J$. La prise en compte des interactions permet alors l'apparition d'un type nouveau d'excitations: les modes collectifs. Ceux-ci apparaissent dès lors que le critère de Stoner dynamique est satisfait :

$$
1-V(\vec{q}) \operatorname{Re} \chi_{0}(\vec{q}, \omega)=0
$$

Les pôles $\left(\vec{q}_{i}, \omega_{i}\right)$ satisfaisant l'Eq. (3.13) pour des énergies inférieures à $\omega_{c}(\vec{q})$, i.e. où $\operatorname{Im} \chi_{0}(\vec{q}, \omega)=0$, correspondent à des divergences de la partie imaginaire de la susceptibilité magnétique qui pourraient être à l'origine des excitations magnétiques mesurées par diffusion des neutrons. Les autres pôles situés dans le continuum pour des énergies supérieures à $\omega_{c}(\vec{q})$, i.e. où $\operatorname{Im} \chi_{0}(\vec{q}, \omega) \neq 0$, sont amortis par les excitations el-t. Dans les cuprates, du fait de la symétrie du gap supraconducteur et donc de la forme de $\omega_{c}(\vec{q})$, les modes collectifs sont confinés dans un petit espace en vecteur d'onde et en énergie autour du vecteur d'onde AF dans les régions «gappées » montrées sur la Fig. 16. C'est précisément ce qui est observé expérimentalement (Fig. 17) et confirmé par les calculs numériques de la susceptibilité RPA dans l'état supraconducteur. Nous montrons deux exemples de calcul sur la Fig. 18 réalisés pour $\mathrm{Bi}_{2} \mathrm{Sr}_{2} \mathrm{CaCu}_{2} \mathrm{O}_{8+\delta}$ et $\mathrm{YBa}_{2} \mathrm{Cu}_{3} \mathrm{O}_{7}$ à partir de la structure de bande et du gap supraconducteur mesurés par ARPES. Les calculs rendent parfaitement compte de l'apparition d'un spectre magnétique centré au vecteur d'onde $\mathrm{AF}$ avec une structure similaire à celle mesurée expérimentalement.

La partie imaginaire ${ }^{2}$ RPA de l'exciton de spin le long de sa dispersion, $\omega_{r}(\vec{q})$, s'écrit (volume 2 p. 77) :

$$
\operatorname{Im} \chi(\vec{q}, \omega)_{R P A}=Z(\vec{q}) \delta\left(\hbar \omega-\hbar \omega_{r}(\vec{q})\right)
$$

\footnotetext{
${ }^{2}$ La partie réelle se déduit de la partie imaginaire à partir des relations de Kramers-Kronig, $\operatorname{Re} \chi(\vec{q}, \omega)_{R P A}=$ $\frac{1}{\pi} \mathcal{P} \int_{-\infty}^{\infty} d \omega^{\prime} \frac{\operatorname{Im} \gamma(\vec{q}, \omega)_{R P A}}{\omega^{\prime}-\omega}$, nous obtenons :
}

$$
\operatorname{Re\chi }(\vec{q}, \omega)_{R P A} \sim-\frac{1}{\pi \hbar} \frac{Z(\vec{q})}{\omega-\omega_{r}(\vec{q})} .
$$



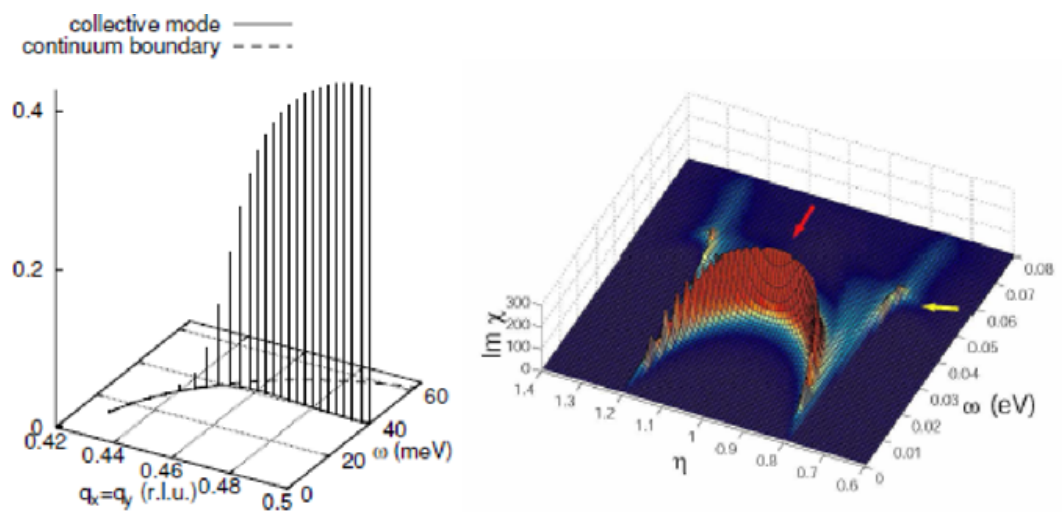

Figure 18. Calcul numérique de la susceptibilité magnétique en interaction dans l'état supraconducteur [14,20]. (gauche) Dispersion et intensité des modes collectifs suivant la direction (110) calculées à partir des données d'ARPES de $\mathrm{Bi}_{2} \mathrm{Sr}_{2} \mathrm{CaCu}_{2} \mathrm{O}_{8+\delta}$. La bordure du continuum est représentée par la ligne pointillée. (droite) Identique à la figure de gauche mais en utilisant des paramètres pour reproduire le spectre expérimentale, montré sur la Fig Fig-ResoDisp, dans le système $\mathrm{YBa}_{2} \mathrm{Cu}_{3} \mathrm{O}_{7}$.

où $Z(\vec{q})$ correspond au poids spectral du mode qui, en couplant les Eq. (3.14) et Eq. (3.12), s'exprime par :

$$
Z(\vec{q})=\frac{\pi}{V(\vec{q})^{2}}\left[\frac{\partial}{\partial \omega} \operatorname{Re} \chi^{0}(\vec{q}, \omega)\right]_{\omega=\omega_{r}}^{-1}
$$

Le poids spectral de l'exciton de spin dépend donc du comportement divergent de la partie réelle de la susceptibilité. Or, dans l'état supraconducteur, $\operatorname{Im} \chi_{0}(\vec{q}, \omega)$ est une fonction "marche", nulle jusqu'à l'énergie seuil du continuum d'excitation $e-t, \omega_{c}(\vec{q})$. Il en résulte une divergence de la partie réelle. Plusieurs comportements sont possibles en fonction du dopage et de la topologie de la surface de Fermi. Au dopage optimum du diagramme de phase, où la surface de Fermi est ouverte, la divergence est de type logarithmique $: \operatorname{Re} \chi_{0}\left(q_{A F}, \omega\right) \propto \log 1 /\left(\omega_{c}\left(q_{A F}\right)-\omega\right)$. En conséquence, une excitation collective, $\omega_{r}$, apparaît comme un état lié au continuum d'excitation $e l-t$ dont le poids spectral est proportionnel à son énergie de liaison réduite : $\omega_{c}(\vec{q})-\omega_{r}(\vec{q})$. Ainsi $Z(\vec{q})$ s'écrit :

$$
Z(\vec{q}) \propto \frac{\pi}{V(\vec{q})^{2}} \frac{\omega_{c}(\vec{q})-\omega_{r}(\vec{q})}{\omega_{c}(\vec{q})}
$$

Ainsi, plus l'excitation collective (ou état lié) est proche du continuum d'excitations el- $t$ et plus son intensité est faible.

\subsection{Modèle itinérant dans un systéme à 2 plans $\mathrm{CuO}_{2}$ par maille élémentaire}

Dans $\mathrm{YBa}_{2} \mathrm{Cu}_{3} \mathrm{O}_{7-\delta}$, la distance inter-plan est d'environ $3 \AA$, donc plus petite que la distance $\mathrm{Cu}-\mathrm{Cu}$ dans le plan $\mathrm{CuO}_{2}$. Il est donc nécessaire de considérer les couplages entre ces deux plans pour la modélisation. Comme nous l'avons précisé plus haut, le couplage interplan conduit à une susceptibilité acoustique $(a c)$ et optique $(o p)$. Dans un modèle itinérant, il y a deux facons de considérer l'origine du couplage entre les deux plans $\mathrm{CuO}_{2}$. Soit on suppose un couplage électronique cohérent entre les plans conduisant à deux bandes électroniques liantes et antiliantes, soit on introduit un terme d'échange magnétique de plan à plan, ou encore les deux. Expérimentalement, par ARPES, il est clairement observé deux bandes, liante et antiliante et, par neutron, un couplage magnétique perpendiculaire aux plans $\mathrm{CuO}_{2}$ dans la phase $\mathrm{AF}$ isolante à dopage nul. Pour simplifier, nous considérons néanmoins le cas d'une seule bande et d'un couplage magnétique suivant l'axe $\vec{c}, \mathrm{~J}_{\perp}$. L'interaction $I(\vec{q})$ possède également 
2 composantes ac et $o p$ :

$$
\begin{gathered}
I_{\vec{q}}^{a c}=I_{\vec{q}}+I_{\perp} \\
I_{\vec{q}}^{o p}=I_{\vec{q}}-I_{\perp}
\end{gathered}
$$

Les susceptibilités RPA dans les canaux $a c$ et $o p$ s'écrivent :

$$
\begin{aligned}
& \chi_{a c}(\omega, \vec{q})=\frac{\chi^{0}(\omega, \vec{q})}{1-V_{\vec{q}}^{a c} \chi^{0}(\omega, \vec{q})} \\
& \chi_{o p}(\omega, \vec{q})=\frac{\chi^{0}(\omega, \vec{q})}{1-V_{\vec{q}}^{o p} \chi^{0}(\omega, \vec{q})}
\end{aligned}
$$

où $V(\vec{q})^{a c, o p}=\frac{2 I(\vec{q})^{a c, o p}}{\left(g \mu_{B}\right)^{2}}$.

Des excitons de spins apparaissent dans chacun des canaux pour des couples $\left(\vec{q}_{i}, \omega_{i}\right)$ qui satisfont les critères de Stoner dynamiques : $1-V(\vec{q})^{a c, o p} \operatorname{Re} \chi_{0}(\vec{q}, \omega)=0$. Nous montrons sur la Fig. 19 de gauche les calculs numériques des parties réelles $a c$ et $o p$ de la susceptibilité sans interaction, $\operatorname{Re} \chi_{0}\left(q_{\vec{A} F}, \omega\right)$, en indiquant par des doubles flèches les positions en énergie satisfaisant les critères de Stoner dynamiques. Du fait de $I_{\perp}$, l'interaction $a c$ est amplifiée, voir Eq. (3.18), impliquant des énergies de liaisons différentes des excitons de spins dans les canaux acoustique et optique : $\left(\omega_{c}(\vec{q})-\omega_{r}^{a c}(\vec{q})\right)>\left(\omega_{c}(\vec{q})-\omega_{r}^{o p}(\vec{q})\right)$. En conséquence, en prenant le cas à $\mathrm{q}=\mathrm{q}_{A F}$, l'exciton de spin dans le canal acoustique apparaît à plus basse énergie et avec une plus grande intensité que l'excition de spin dans le canal optique, voir Fig. 19. Le modèle itinérant donne donc une explication simple de la différence d'intensité observée entre les modes $a c$ et op, voir Fig. 12.

Ainsi, à partir des énergies des modes $a c$ et $o p$ et du rapport de leurs poids spectraux mesurés par neutron, $\alpha=W_{r}^{a c} / W_{r}^{o p}$, il est possible, à partir de l'Eq. (3.17), d'estimer la bordure du continuum :

$$
\omega_{c}\left(\vec{q}_{A F}\right)=\frac{E_{r}^{o p} \alpha-E_{r}^{a c}}{\alpha-1} .
$$
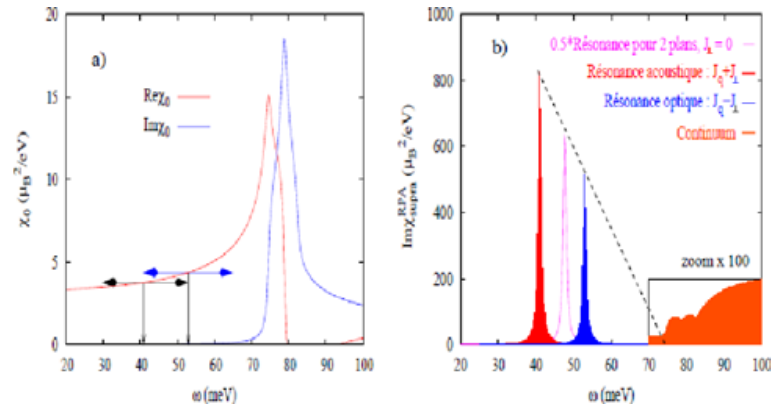

Figure 19. Résonances acoustique/optique et extrapolation de la bordure du continuum d'excitation $e l-t$ à $\vec{q}_{A F}$, $\omega_{c}\left(\vec{q}_{A F}\right)$ [57]. (gauche) nous avons représenté en rouge la partie réelle et en bleu la partie imaginaire de la susceptibilité transverse sans interaction dans l'état supraconducteur. Les doubles flèches noires et bleues indiquent les valeurs $\frac{1}{V\left(\vec{q}_{A F}\right)^{a c}}$ et $\frac{1}{V\left(\vec{q}_{A F}\right)^{o p}}$ correspondantes aux conditions de pôles au vecteur d'onde AF dans les canaux acoustique et optique (Eq. (3.20)) (droite) calcul numérique de $\operatorname{Im} \chi_{a c}(\omega, \vec{q})$ en bleue et $\operatorname{Im} \chi_{o p}(\omega, \vec{q})$ optique en bleu. En rouge, nous montrons le calcul pour un biplan non couplé, $I_{\perp}=0$. Dans l'insert, en bas à droite, nous avons zoomé, à partir de $70 \mathrm{meV}$, la bordure du continuum d'excitations électron-trou, $\omega_{c}\left(\vec{q}_{A F}\right)$. La ligne pointillée noire montre que par une simple extrapolation linéaire, il est possible d'estimer la bordure du continuum au vecteur d'onde AF. 


\section{CONCLUSION}

Nous espérons avoir montré tout au long de cet article que les mesures de diffusion inélastique des neutrons ont apporté, et continuent de le faire, une contribution décisive à l'étude des mécanismes de la supraconductivité non conventionnelle. Dans l'état supraconducteur des cuprates, ces mesures ont permis d'identifier l'apparition de tout un mode magnétique centré au vecteur d'onde antiferromagnétique et ayant une fréquence caractéristique de $40 \mathrm{meV}$ (soit $9.8 \mathrm{THz}$ ) et dont la dispersion en fonction du vecteur d'onde forme une structure étrange en forme de X.

Nous avons vu que lorsqu'ils ne sont ni isolants ni supraconducteurs, les cuprates sont de mauvais métaux, très mal décrits par le modèle du liquide de Fermi traditionnel. Le mouvement des électrons y est fortement contraint par le magnétisme formé par leurs spins. Cette dualité entre les caractères itinérant/localisé des électrons est sans doute à l'origine de la complexité intrinsèque de cet état particulier. Faut-il alors traiter ces fortes corrélations comme une perturbation d'un état métallique classique en prenant en compte une interaction effective entre charges qui inclut le couplage avec les spins ? Ou est-ce que de nouvelles organisations électroniques sont à considérer ? Dans tous les cas, il apparaît essentiel de pouvoir sonder l'environnement magnétique, ainsi que la manière dont celui-ci se déforme dans le temps, pour comprendre le milieu dans lequel évoluent les charges. La diffusion des neutrons est l'une des sondes les mieux adaptées permettant d'explorer dans tout l'espace réciproque les propriétés magnétiques statique et dynamique des cuprates, et a fortiori des systèmes physiques présentant de fortes corrélations.

\section{Références}

[1] A. Fetter and J. Walecka. Quantum Theory of Many-Particle Systems. Dover Publications, 2003.

[2] A.S. Alexandrov. Theory of superconductivity. Institute of Physics (IOP), 2004.

[3] E. Altendorf, X.K. Chen, J.C. Irwin, R. Liang, and W.N. Hardy. Temperature dependences of the 340-, 440-, and 500-cm-1 raman modes of yba2cu3oy for $\mathrm{y}=6.7$ and $\mathrm{y}=7.0$. Phys. Rev. B, 47:8140 $-8150,1993$.

[4] M. Arai, T. Nishijima, Y. Endoh, T. Egami, S. Tajima, K. Tomimoto, Y. Shiohara, M. Takahashi, A. Garrett, and S. M. Bennington. Incommensurate spin dynamics of underdoped superconductor $\mathrm{yba}_{2} \mathrm{cu}_{3} \mathrm{O}_{6.7}$. Phys. Rev. Letters, 83(3):608 - 611, 1997.

[5] P. Bourges. From magnons to the resonance peak: Spin dynamics in high- $t_{c}$ superconducting cuprates by inelastic neutron scattering. In J. Bok, G. deutscher, D. Pavuna, and S.A. Wolf, editors, The gap Symmetry and Fluctuations in High Temperature Superconductors. Plenum Press, 1998.

[6] P. Bourges, H.F. Fong, L.P. Regnault, J. Bossy, C. Vettier, D.L. Milius, and A. Aksay. High-energy spin excitations in $\mathrm{yba}_{2} \mathrm{cu}_{3} \mathrm{O}_{6.5}$. Phys. Rev. B, 56(18):R11439 - R11442, 1997.

[7] P. Bourges, B. Keimer, S. Pailhès, L.P. Regnault, Y. Sidis, and C. Ulrich. The resonant magnetic mode: A common feature of high-tc superconductors. Phyica C, 424(1-2):45 - 49, 2005.

[8] P. Bourges, L.P. Regnault, Y. Sidis, and C. Vettier. Inelastic-neutron-scattering study of antiferromagnetic fluctuations in $\mathrm{yba}_{2} \mathrm{Cu}_{3} \mathrm{O}_{6.97}$. Phys. Rev. B, 53:876 - 885, 1996.

[9] P. Bourges, Y. Sidis, H. F. Fong, L. P. Regnault, J. Bossy, A. Ivanov, and B. Keimer. The spin excitation spectrum in superconducting in $\mathrm{yba}_{2} \mathrm{Cu}_{3} \mathrm{O}_{6.85}$. Science, 288(5469):1234 - 1237, 2000.

[10] N. Bulut and D.J. Sclapino. $\mathrm{d}_{x^{2}-y^{2}}$ symmetry and the pairing mechanism. Phys. Rev. B, 54(21): 14971 - 14973, 1996.

[11] J. C. Campuzano, M. R. Norman, and M. Randeria. Photoemission in the high tc superconductors. In K. H. Bennemann and J. B. Ketterson, editors, Physics of Conventional and Unconventional Superconductors. Springer Berlin, 2004.

[12] L. Capogna, B. Fauqué, Y. Sidis, C. Ulrich, P. Bourges, S. Pailhès, A. Ivanov, J. L. Tallon, B. Liang, C. T. Lin, A. I. Rykov, and B. Keimer. Odd and even magnetic resonant modes in highly overdoped $\mathrm{bi}_{2} \mathrm{sr}_{2} \mathrm{cacu}_{2} \mathrm{O}_{8+\delta}$. Phys. Rev. B, 75:060503(R)1 - 060503(R)4, 2007. 
[13] N.B. Christensen, D.F. McMorrow, H.M. Rønnow, B. Lake, S.M. Hayden, G. Aeppli, T.G. Perring, M. Mangkorntong, M. Nohara, and H. Takagi. Dispersive excitations in the hightemperature superconductor $\mathrm{la}_{2-x} \mathrm{Sr}_{x} \mathrm{cuO}_{4}$. Phys. Rev. Letters, 93(14):147002(1) - 147002(4), 2004.

[14] A.V. Chubukov, B. Janko, and O. Tchernyshyov. Dispersion of the neutron resonance in cuprate superconductors. Phys. Rev. B, 63:180507(R)1 - 180507(R)4, 2001.

[15] R. Coldea, S. M. Hayden, G. Aeppli, T. G. Perring ADN C. D. Frost, T. E. Mason, S.-W. Cheong, and Z. Fisk. Spin waves and electronic interactions in la cuo $_{4}$. Phys. Rev. Letters, 86(23):5377 5380, 2001.

[16] E. Dagotto. Correlated electrons in high-temperature superconductors. Rev. Mod. Phys., 66:763 840, 1994.

[17] Pengcheng Dai, S. M. Hayden, G. Aeppli, T. G. Perring, F. Doan, and H. A. Mook. Spin fluctuations in ybacuo. Nature, 395:580 - 582, 1998.

[18] Dai, H. A. Mook, and R.D. Hunt. Evolution of the resonance and incommensurate spin fluctuations in superconducting $\mathrm{yba}_{2} \mathrm{cu}_{3} \mathrm{O}_{6+x}$. Phys. Rev. B, 63:054525(1)-054525(20), 2001.

[19] A. Damascelli, Z. Hussain, and Z-X. Shen. Angle-resolved photoemission studies of the cuprate superconductors. Rev. Mod. Phys., 75:743 - 541, 2003.

[20] I. Eremin, D. K. Morr, A. V. Chubukov, K. H. Bennemann, and M. R. Norman. Novel neutron resonance mode in dx2-y2-wave superconductors. Phys. Rev. Letters, 94:147001-1 - 147001-4, 1992.

[21] B. Fauqué. Etude des supraconducteurs à haute température critique par diffusion des neutrons. PhD thesis, Université Paris XI, 2007.

[22] B. Fauqué, Y. Sidis, L. Capogna, A. Ivanov, K. Hradil, C. Ulrich, A. I. Rykov, B. Keimer, and P. Bourges. Dispersion of the odd magnetic resonant mode in near-optimally doped bi $_{2} \mathrm{Sr}_{2} \mathrm{cacu}_{2} \mathrm{O}_{8+\delta}$. Phys. Rev. B, 76:214512(1)-214512(15), 2001.

[23] H. F. Fong, P. Bourges, Y. Sidis, L. P. Regnault, A. Ivanov, G. D. Gu, N. Koshizuka, and B. Keimer. Neutron scattering from magnetic excitations in bisrcacuo. Nature, 398:588 - 591, 1999.

[24] H.F. Fong, P. Bourges, Y. Sidis, L.P. Regnault, J. Bossy, A. Ivanov, D.L. Milius, I.A. Aksay, and B. Keimer. Spin susceptibility in underdoped $\mathrm{yba}_{2} \mathrm{cu}_{3} \mathrm{O}_{6+x}$. Phys. Rev. B, 61:14773 - 14786, 2000.

[25] H.F. Fong, B. Keimer, P.W. Anderson, D. Reznik, F. Dogan, and I.A. Aksay. Phonon and magnetic neutron scattering at $41 \mathrm{mev}$ in $\mathrm{yba}_{2} \mathrm{cu}_{3} \mathrm{O}_{7}$. Phys. Rev. Letters, 75:316 - 319, 1995.

[26] H.F. Fong, B. Keimer, D. Reznik, D.L. Milius, and I.A. Aksay. Polarized and unpolarized neutronscattering study of the dynamical spin susceptibility of $\mathrm{yba}_{2} \mathrm{Cu}_{3} \mathrm{O}_{7}$. Phys. Rev. B, 54:6708 - 6720, 1996.

[27] M. Fujita, H. Goka, K. Yamada, J.M. Tranquada, and L.P. Regnault. Stripe order, depinning, and fluctuations in $\mathrm{la}_{1.875} \mathrm{ba}_{0.125} \mathrm{cuo}_{4}$ and $\mathrm{la}_{1.875} \mathrm{ba}_{0.075} \mathrm{Sr}_{0.050} \mathrm{cuo}_{4}$. Phys. Rev. B, 70:104517 - 104528, 2004.

[28] Y. Gallais. Diffusion Raman électronique dans les cuprates supraconducteurs. PhD thesis, Université Pierre et Marie Curie, Paris VI, 2003.

[29] S.M. Hayden, G. Aeppli, T.G. Perring, H.A. Mook, and F. Dogan. High-frequency spin waves in yba $_{2} \mathrm{cu}_{3} \mathrm{O}_{6.15}$. Phys. Rev. B, 54(10):R6905 - R6908, 1996.

[30] H. He, P. Bourges, Y. Sidis, C. Ulrich, L. P. Regnault, S. Pailhès, N. S. Berzigiarova, N. N. Kolesnikov, and B. Keimer. Magnetic resonant mode in the single-layer high-temperature superconductor $\mathrm{tl}_{2} \mathrm{ba}_{2} \mathrm{cuo}_{6+\text { delta }}$. Science, 295(5557):1045 - 1047, 2002.

[31] H. He, Y. Sidis, P. Bourges, G. D. Gu, A. Ivanov, N. Koshizuka, B. Liang, C. T. Lin, L. P. Regnault, E. Schoenherr, and B. Keimer. Resonant spin excitation in an overdoped high temperature superconductor. Phys. Rev. Letters, 86:1610 - 1613, 2001.

[32] V. Hinkov, P. Bourges, S. Pailhès, Y. Sidis, A. Ivanov, C.D. Frost, T.G. Perring, C. T. Lin, D. P. Chen, and B. Keimer. Spin dynamics in the pseudogap state of a high-temperature superconductor. Nature Phys., 3:780 - 785, 2007. 
[33] V. Hinkov, S. Pailhès, P. Bourges, Y. Sidis, A. Ivanov, A. Kulakov, C. T. Lin, D. P. Chen, C. Bernhard, and B. Keimer. Two-dimensional geometry of spin excitations in the high-transitiontemperature superconductor $\mathrm{yba}_{2} \mathrm{Cu}_{3} \mathrm{O}_{6+x}$. Nature, 430:650 - 653, 2004.

[34] S. Hufner. Photoelectron spectroscopy. Principles and application. Springer-Verlag Berlin and Heidelberg GmbH, 2003.

[35] M. Imada, A. Fujimori, and Y. Tokura. Metal-insulator transition. Rev. Mod. Phys., 70(4):1039 $1263,1998$.

[36] A. Kaminski, M. Randeria, J.C. Campuzano, M.R. Norman, H. Fretwell, J. Mesot, T. Sato, T. Takahashi, and K. Kadowaki. Renormalization of spectral line shape and dispersion below tc in $\mathrm{bi}_{2} \mathrm{Sr}_{2} \mathrm{Cacu}_{2} \mathrm{O}_{8+\delta}$. Phys. Rev. Letters, 86(6):1070 - 1074, 2001.

[37] C. Khalin, M.R. Norman, and D. Pines. The pseudogap : friend or foe of high tc. Adv. Phys., 54(8):715 - 733, 1999.

[38] S. A. Kivelson, I. P. Bindloss, E. Fradkin, V. Oganesyan, J. M. Tranquada, A. Kapitulnik, and C. Howald. How to detect fluctuating stripes in the high-temperature superconductors. Rev. Mod. Phys., 75(4):1201 - 1241, 2003.

[39] S.A. Kivelson and E. Fradkin. How optimal inhomogeneity produces high temperature superconductivity. In J.R. Schrieffer and J.S. Brooks, editors, Handbook of High-Temperature Superconductivity Theory and Experiment. Springer New York, 2007.

[40] A. A. Kordyuk, S. V. Borisenko, M. S. Golden, S. Legner, K. A. Nenkov, M. Knupfer, J. Fink, H. Berger, L. Forró, and R. Follath. Doping dependence of the fermi surface in (bi,pb) ${ }_{2} \mathrm{sr}_{2} \mathrm{cacu}_{2} \mathrm{O}_{8+\delta}$. Phys. Rev. B, 66:014504(1)-014504(6), 2002.

[41] A. A. Kordyuk, S. V. Borisenko, A. Koitzsch, J. Fink, M. Knupfer, and H. Berger. Bare electron dispersion from experiment: Self-consistent self energy analysis of photoemission data. Phys. Rev. B, 71:214513(1) - 214513(11), 2005.

[42] M. Lavagna and G. Stemman. Spin excitations of two-dimensional-lattice electrons: Discussion of neutron-scattering and nmr experiments in high-tc superconductors. Phys. Rev. B, 49:4235 4250, 1994.

[43] P. Lee, N. Nagaosa, and X.G. Wen. Doping a mott insulator: Physics of high-temperature superconductivity. Rev. Mod. Phys., 78:17 - 84, 2006.

[44] S.W. Lovesey. Theory of neutron scattering from condensed matter, volume 1-2. Oxford Science Publication, 1986.

[45] Jian Ping Lu. Neutron scattering as a probe for unconventionnal supercondcuting states. Phys. Rev. Letters, 68(1):125 - 129, 1992.

[46] D. Manske. Theory of unconventionnal superconductors. Cooper pairing mediated by spin excitations. Spinger, 2006.

[47] W. Meevasana, F. Baumberger, K. Tanaka, F. Schmidt, W.R. Dunkel, D.H. Lu, S.-K. Mo, H. Eisaki, and Z.X. Shen. Extracting the spectral function of the cuprates by a full two-dimensional analysis: Angle-resolved photoemission spectra of $\mathrm{bi}_{2} \mathrm{sr}_{2} \mathrm{cuo}_{6}$. Phys. Rev. B, 77:104506(1) - 104506(7), 2008.

[48] J. Mesot, M. R. Norman, H. Ding, M. Randeria, J. C. Campuzano, A. Paramekanti, H. M. Fretwell, A. Kaminski, T. Takeuchi, T. Yokoya, T. Sato, T. Takahashi, T. Mochiku, and K. Kadowaki. Superconducting gap anisotropy and quasiparticle interactions: A doping dependent photoemission study. Phys. Rev. Letters, 83:840 - 843, 1999.

[49] V.P. Mineev and K. Samokhin. Introduction to unconventionnal superconductivity. Gordon and Breach Science Publisher, 1999.

[50] H.A. Mook, M. Yethiraj, G. Aeppli, T.E. Mason, and T. Armstrong. Polarized neutron determination of the magnetic excitations in $\mathrm{yba}_{2} \mathrm{Cu}_{3} \mathrm{O}_{7}$. Phys. Rev. Letters, 70:3490 - 3493, 1993.

[51] M.R. Norman. Linear response theory and the universal nature of the magnetic excitation spectrum of the cuprates. Phys. Rev. B, 75:184514-184527, 2007.

[52] M.R. Norman and C.Pt'epin. The electronic nature of high temperature cuprate supeconductors. Rep. Prog. Phys., 66:1547 - 1610, 2003. 
[53] M.R. Norman, H. Ding, H. Fretwell, M. Randeria, and J.C. Campuzano. Extraction of the electron self-energy from angle-resolved photoemission data: Application to $\mathrm{bi}_{2} \mathrm{Sr}_{2} \mathrm{Cacu}_{2} \mathrm{O}_{8+x}$. Phys. Rev. $B, 60(10): 7585$ - 7589, 1999.

[54] M. Okawa, K. Ishizaka, H. Uchiyama, H. Tadatomo, T. Masui, S. Tajima, X.-Y. Wang, C.-T. Chen, S. Watanabe, A. Chainani, T. Saitoh, and S. Shin. Superconducting electronic state in optimally doped $\mathrm{yba}_{2} \mathrm{Cu}_{3} \mathrm{O}_{7-\delta}$ observed with laser-excited angle-resolved photoemission spectroscopy. Phys. Rev. B, 79:144528(1) - 144528(9), 2009.

[55] F. Onufrieva and P. Pfeuty. Quantum critical point associated with the electronic topological transition in a two-dimensional electron system as a driving force for anomalies in underdoped high-tc cuprates. Phys. Rev. B, 61(1):799 - 820, 2000.

[56] F. Onufrieva and P. Pfeuty. Spin dynamics of a two-dimensional metal in a superconducting state: Application to the high-tc cuprates. Phys. Rev. B, 65(1):054515(1) - 054515(11), 2002.

[57] S. Pailhès. Etude des excitations magnétiques dans le supraconducteur à haute température critique $\mathrm{Y}_{1-x} \mathrm{Ca}_{x} \mathrm{Ba}_{2} \mathrm{Cu}_{3} \mathrm{O}_{7-\delta}$. PhD thesis, Université Pierre et Marie Curie, Paris VI, 2004.

[58] S. Pailhès, Y. Sidis, P. Bourges, V. Hinkov, A. Ivanov, C. Ulrich, L. P. Regnault, and B. Keimer. Resonant magnetic excitations at high energy in superconducting $\mathrm{yba}_{2} \mathrm{cu}_{3} \mathrm{O}_{6.85}$. Phys. Rev. Letters, 93:167001, 2004.

[59] S. Pailhès, Y. Sidis, P. Bourges, C. Ulrich, V. Hinkov, L. P. Regnault, A. Ivanov, B. Liang, C. T. Lin, C. Bernhard, and B. Keimer. Two resonant magnetic modes in an overdoped high tc superconductor. Phys. Rev. Letters, 91:237002, 2003.

[60] M. Randeria, A. Paramekanti, and N. Trivedi. Nodal quasiparticle dispersion in strongly correlated d-wave superconductors. Phys. Rev. B, 69:144509(1) - 144509(5), 2004.

[61] D. Reznik, P. Bourges, H.F. Fong, L.P. Regnault, J. Bossy, C. Vettier, D.L. Milius, I.A. Aksay, and B. Keimer. Direct observation of optical magnons in $\mathrm{yba}_{2} \mathrm{cu}_{3} \mathrm{O}_{6.2}$. Phys. Rev. B, 53(22):R14741 R14744, 1996.

[62] J. Rossat-Mignod, L.P. Regnault, C. Vettier, P. Bourges, P. Burlet, and J. Bossy. Neutron-scattering study of the $\mathrm{yba}_{2} \mathrm{Cu}_{3} \mathrm{O}_{6+x}$ system. Physica $C, 185: 86-92,1991$.

[63] J. Robert Schrieffer and S. Brooks. Handbook of High-Temperature Superconductivity Theory and Experiment, volume 1-2. Springer New York, 2007.

[64] P. Schweiss, W. Reichardt, M. Braden, G. Collin, G. Heger, H. Claus, and A. Erb. Static and dynamic displacements in $\mathrm{rba}_{2} \mathrm{Cu}_{3} \mathrm{O}_{7-\delta}(\mathrm{r}=\mathrm{y}, \mathrm{ho} ; \delta=0.05,0.5)$ : A neutron-diffraction study on single crystals. Phys. Rev. B, 49:1387 - 1396, 1994.

[65] S. Shamoto, M. Sato, J. M. Tranquada, B. J. Sternlieb, and G. Shirane. Neutron-scattering study of antiferromagnetism in $\mathrm{yba}_{2} \mathrm{cu}_{3} \mathrm{O}_{6.15}$. Phys. Rev. B, 48:13817-13825, 1993.

[66] M. Le Tacon. Un gap peut en cacher un autre: exploration. Une exploration de la phase supraconductrice des cuprates par sonde Raman électronique. $\mathrm{PhD}$ thesis, Université Pierre et Marie Curie, Paris VII, 2006.

[67] J.L. Tallon, T. Benseman, C.V.M. Williams, and J.W. Loram. The phase diagram of high-tc superconductors. Physica C, 415(1-1):9-14, 2004.

[68] T. Timusk and B. Statt. The pseudogap in high-temperature superconductors : an experiemental survey. Rep. Prog. Phys., 62(8):61 - 122, 1999.

[69] J. M. Tranquada, B. J. Sternlieb, J. D. Axe, Y. Nakamura, and S. Uchida. Evidence for stripe correlations of spins and holes in copper oxide superconductors. Nature, 375:561 - 563, 1995.

[70] J. F. Zasadzinski, L. Ozyuzer, N. Miyakawa, K. E. Gray, D. G. Hinks, and C. Kendziora. Correlation of tunneling spectra in $\mathrm{bi}_{2} \mathrm{Sr}_{2} \mathrm{Cacu}_{2} \mathrm{O}_{8+\delta}$ with the resonance spin excitation. Phys. Rev. Letters, 87:067005(1) - 067005(4), 2001.

[71] F. C. Zhang and T. M. Rice. Effective hamiltonian for the superconducting cu oxides. Phys. Rev. $B, 37: 3759-3761,1988$. 